Euskal ikerketen aldizkaria | Revue d'études basques |

Revista de estudios vascos | Basque studies review

$5 \mid 2000$

Numéro V

\title{
À propos d'une construction non-standard du basque
}

\section{Georges Rebuschi}

\section{OpenEdition}

Journals

Édition électronique

URL : http://journals.openedition.org/lapurdum/1318

DOI : 10.4000/lapurdum.1318

ISSN : 1965-0655

Éditeur

IKER

Édition imprimée

Date de publication : 1 octobre 2000

Pagination : 237-282

ISBN : 2-84127-161-7

ISSN : 1273-3830

Référence électronique

Georges Rebuschi, « À propos d'une construction non-standard du basque », Lapurdum [En ligne], 5 2000, mis en ligne le 01 juin 2009, consulté le 04 juillet 2020. URL : http://journals.openedition.org/ lapurdum/1318; DOI : https://doi.org/10.4000/lapurdum.1318 
Georges REBUSCHI

\section{A propos d'une construction non-standard du basque}

\section{Lapurdum 5 [Bayonne]}

(2000)

pp. $237-282$ 


\section{A PROPOS D'UNE CONSTRUCTION}

NON-STANDARD DU BASQUE

\section{Plan}

1. Introduction 1

2. Les relatives libres et semi-libres du basque 2

3. Le cadre et les enjeux syntaxiques 6

4. Vers une solution unitaire 9

5. RSL elliptiques et non-elliptiques 13

6. Un corrélat sémantique fondamental 15

$\begin{array}{ll}\text { 7. Résumé et conclusions } & 17\end{array}$

Appendice $\quad 19$

Références $\quad 35$

\section{Introduction}

Depuis La grammaire des fautes de Frei (1929), on sait que certains écarts par rapport à la langue normalisée sont systématiques, donc significatifs et révélateurs de potentialités de la langue ignorées par sa version «académique». De ce point de vue, dans le cadre de la linguistique moderne et formelle, on peut citer entre autres deux exemples qui se sont révélés particulièrement féconds: d'une part, les débats autour de la contraction de la séquence anglaise want to 'vouloir + INFINITIF" en "wanna", rejetée par tous les prescriptivistes, mais dont la distribution est très clairement contrainte syntaxiquement; d'autre part, certains travaux sur l'accord du participe passé en français spontané, qui est soit optionnel, soit tout simplement bloqué en fonction du contexte dans lequel le participe apparaît. Je voudrais étudier ici dans cet esprit une tournure qui est rejetée par tous les bascophones que je connais, mais qui est pourtant attestée dans tous les dialectes dans des textes des $18^{\mathrm{e}}$ et $19^{\mathrm{e}}$ siècles $^{*}$. Il s'agit du fait que les "relatives semi-libres" (cf. 2.2 pour des détails), comme la séquence entre crochets de (1a), lorsqu'elles sont disloquées, c'est-à-dire topicalisées et séparées du reste de la phrase par une pause, voire reprises par un pronom explicite, peuvent porter la marque ergative qui correspond à la position relativisée à l'intérieur de la relative, plutôt qu'à la fonction de l'expression entière dans la phrase matrice, $\mathrm{cf}$. (1b), où le suffixe ergatif «fautif» est en gras et précédé du symbole "ڤ":

(1) a (basque standard)

[Huts eginen du.en.Ø.a] (, hura) gaztigatua izanen da.

faute faire-FUT AUX-en-Ø-SG(-ABS) lui puni-SG être-FUT AUX ${ }^{1}$

'Celui qui [lit. le qui] commettra une erreur (, celui-là) sera puni.'

\footnotetext{
* Ce travail reprend, en en amplifiant la partie descriptive, la première partie de mon exposé (Rebuschi 2000) : je remercie l'audience de cette Table ronde pour ses remarques, en particulier Henk van Riemsdijk et Alex Grosu, ainsi qu'A. Lacombe pour avoir attiré mon attention, il y a bien plus longtemps, sur la bizarrerie factuelle que constitue la coordination non-additive des DP étudiée en 6.2 (cf. l'ex. (29)) (le phénomène global traité ici était simplement signalé de manière anecdotique dans la section 6 de Rebuschi (sous presse), rédigé au printemps 1999).

${ }^{1}$ Les abréviations utilisées sont les suivantes:

AUX, auxiliaire ; D, D ${ }^{\circ}$, Dét, déterminant ; DAT, datif; DP, expression nominale (ou syntagme déterminant); ERG, ergatif; FVF, forme verbale fléchie ; FUT, futur ; GEN, génitif; INAC, inaccusatif; INTR, intransitif; MED-P, médio-passif; NEG, négation; NP, syntagme nominal sans déterminant ; PASS, passif; PL,
} 
b (basque non-standard)

[Huts egiten du.en.Ø. $\left.a^{\vartheta} \boldsymbol{k}\right]$, (hura) gaztigatua izanen da. AUX-en-Ø-SG-ERG

On verra qu'un traitement cohérent de cette construction conduit à une hypothèse très simple, et tout à fait compatible à la fois avec la théorie X-barre classique (ou ses avatars minimalistes plus récents), et avec l'hypothèse $\mathrm{DP}$, en faisant crucialement appel à l'activation de la position du spécificateur du DP : cette apparente percolation vers le haut de la marque d'ergatif n'est en effet que la marque phoniquement visible d'une opération beaucoup plus générale en basque, opération qui permet, du côté de la face sémantique des phrases, d'interpréter les RSL comme de simples prédicats plutôt que comme des individus maximas ou des quantificateurs généralisés.

\section{Les relatives libres et semi-libres du basque}

2.1. Les propositions relatives sans antécédent sont au moins de deux types à travers les langues $^{2}$. Il y a d'une part celles qui, du moins superficiellement, se présentent comme de simples propositions, introduites par un pronom relatif ou interrogatif; ce type est illustré pour le français (archaïsant) en (2a), pour l'anglais en (2b), pour l'allemand en (2c), puis pour le basque navarro-labourdin - et souletin, à la morpho-phonologie près - en ( $3 a)$ et sud-oriental en ( $3 b)$, la différence se situant ici au niveau du choix du complémenteur ou marque de subordination, baitau nord, et $-(e) n$ au sud.

(2) a [Qui vivra] verra.

b [Whoever says so] is a liar.

qui-jamais dit ainsi est un menteur

'Quiconque dit de telles choses est un menteur.'

c [Wovon man nicht sprechen kann], daruber muss man schweigen. de-quoi on NEG parler peut, sur-ça doit on se-taire 'Ce dont on ne peut parler, il faut le taire.' [L. Wittgenstein, Tractatus, 1920]

(3) a [Nork ere hutseginen bait.d $\left.u^{3}\right]$, hura gaztigatua izanen da qui-ERG même faute faire-FUT $\mathrm{C}^{\circ}$-AUX lui puni-SG être-FUT AUX lit. 'Qui commettra une faute, (et) celui-là sera puni'

b [Nork ere huts egingo du.en], hura gaztigatua izango da. (id.)

Il est d'usage d'appeler les séquences entre crochets ci-dessus relatives libres, indépendamment du fait qu'elles se trouvent en position argumentale, comme en $(2 a, b)$ ou non, comme en $(2 \mathrm{c})$ et $(3 \mathrm{a}, \mathrm{b})$ - où elles sont disloquées à gauche, et reprises par un pronom (darüber en allemand, et hura en basque ici). ${ }^{4}$

pluriel ; RL, relative libre; RSL, relative semi-libre ; RSL nst., relative semi-libre non-standard; SG, singulier; TR, transitif.

Par ailleurs, le symbole $Ø$ représente pour le moment la " tête nominale" absente ou du moins invisible dans le DP. Pour ne pas induire d'erreur dans l'interprétation de ce symbole, je traiterai l'absolutif comme l'absence de toute marque casuelle, plutôt que comme un nouveau 'zéro', mais insérerai la glose (-ABS) dans les traductions mot-à-mot pour plus de clarté.

2 Je laisse de côté les constructions sémantiquement équivalentes dans les langues qui offrent des relatives dites à tête interne (japonais, coréen, entre autres) : lorsque cette tête est phoniquement nulle, la structure est peut-être encore différente, car elle peut être considérée comme restant in situ, tout comme les têtes lexicales; voir cependant Shimoyama (1997) et Grosu (2000) pour une interprétation qui pourrait bien se ramener au second type décrit infra dans le texte.

${ }^{3}$ Les formes en bait- $+d$-comme bait.da, bait.du, aujourd'hui écrites en deux mots, sont traditionnellement écrites en un, le plus souvent baita, baitu, mais parfois avec un - $d$ - au milieu.

${ }^{4}$ Quand il y a dislocation à gauche, et (éventuelle) reprise par un pronom, on parle souvent de constructions corrélatives pour les phrases complexes entières (cf. Euskaltzaindia 1999, 247-259, pour une présentation générale en basque), et aussi de protase corrélative pour la relative elle-même. Mais ces constructions ne se limitent pas forcément à des tours sans "tête" nominale, où la relative serait libre : la protase peut souvent contenir (même si ce n'est pas le cas en basque) ce qui sera un antécédent (externe) 
2.2. L'autre sorte de relatives sans antécédent est d'apparence plus "nominale", en ce sens qu'un morphème essentiel aux expressions nominales complètes, une marque de nombre et/ou de détermination, y apparaît. C'est ce qu'illustre la séquence entre crochets de (1a), commune à tous les dialectes, où le complémenteur-(e)n est suivi de la marque de nombre et/ou de détermination, - a. C'est ce second type, baptisé relative semi-libre (dorénavant RSL), qui va faire l'objet de ce travail ${ }^{5}$.

Ce type très simple à décrire. Depuis au moins Oyharçabal (1987), on analyse ce que j'appelle ici RSL comme des relatives ordinaires, à ceci près que leur antécédent (ou plutôt «postcédent », pour reprendre l'expression de Lafitte 1962), est, au moins phoniquement, absent. Considérons ainsi la phrase (4):

\section{(4) Gizon.a.k huts egin.en du.}

homme-SG-ERG faute faire-FUT AUX

'L'homme commettra une erreur.'

La position sujet (entre autres) peut être relativisée : superficiellement, il suffit de supprimer le DP correspondant, et de suffixer le complémenteur ou $\mathrm{C}^{\circ}-(e) n$ au verbe fléchi final - en fait, c'est l'aux. qui monte en $\mathrm{C}^{\circ}$, bien entendu; on obtient alors une proposition relative comme (5a), analysée en (5b) - où l'aux. n'est pas déplacé, pour plus de clarté -, proposition qui pourra être associée à un nom qui la suit cf. $(5 \mathrm{c})$, ou, plus rarement, la précède, cf. $(5 \mathrm{~d})^{6}$ :

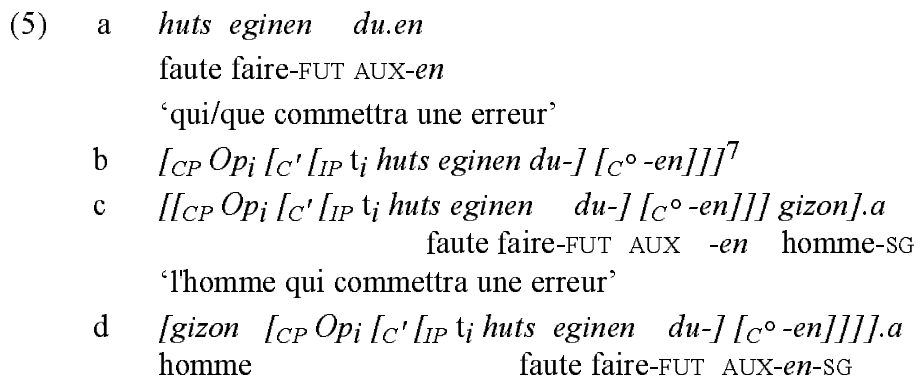

dans la traduction française, et fonctionner comme une restrictive, comme dans l'ex. latin suivant (Gason et al. 1962):

(a)
Quas scripsisti litteras,
quel[ACC-FEM-PL] tu-écrivis lettre[ACC-FEM-PL]
eae mihi joncundissimae fuerunt
elles à-moi très-agréable ont-été
'La lettre que tu as écrite m'a fait grand plaisir'

Il s'ensuit que la question des constructions corrélatives et celle des relatives sans antécédent sont, du point de vue de la linguistique générale, partiellement disjointes.

5 Les "relatives à tête légère" (anglais Light Headed Relatives) du polonais, telles qu'elles sont décrites par Citko (sous presse), et qui sont introduites par un démonstratif qui, crucialement pour sa démonstration, peut aussi jouer un rôle de copule, sont en fait plutôt du type des protases corrélatives lorsqu'elles sont disloquées, si bien que je préfère ne pas employer ce terme pour décrire les données pertinentes du basque. Voir de plus Rebuschi (1998b) pour une description des RL strictes de cette langue partiellement équivalente à celle de Citko, au moins en qui concerne l'hypothèse d'un déplacement de la RL vers la gauche du pronom dit "résomptif".

${ }^{6}$ Cf. en particulier Oyharçabal (1987), et plus récemment Mujika (1999), pour une défense et illustration panchronique et pandialectale de cet ordre des mots (voir aussi par ex. (A20) dans l'Appendice. En tout état de cause, il faut éviter de confondre ce tour avec les structures (superficiellement) appositives comme (i) ci-dessous, dans lesquelles la marque de SG - $a$ est dupliquée, apparaissant d'abord sur l'antécédent, puis sur la relative, qui est alors indiscutablement appositive du point de vue morpho-syntaxique :

(i) gizon.a [huts eginen du.en.a]

Ces dernières structures seront reprises au $\S 6.1$, cf. (25).

${ }^{7}$ Cf. Oyharçabal (1988) pour la première analyse des relatives basques utilisant un opérateur vide, noté "Op" ici. 
A partir de là, et de façon pré-théorique, on peut décrire la construction des RSL comme consistant en l'absence de tout antécédent ou postcédent explicite, cf. la partie pertinente de (1a), reprise ici comme (6), vs. $(5 \mathrm{c})^{8}$ :

(6) $\quad\left[\Gamma_{C P} O p_{i}\left[C^{\prime}\left[_{I P} \mathrm{t}_{i}\right.\right.\right.$ huts eginen $\left.\left.\left.\left.d u-\right]\left[C^{\circ}-e n\right]\right]\right]-\emptyset\right]-a$

faute faire-FUT AUX-en- $\varnothing$-SG

'celui qui commettra une erreur', lit. 'le Ø qui...'

2.3. Deux faits importants pour la suite sont à signaler pour terminer cette présentation générale. Tout d'abord, le symbole " $\varnothing$ " de (6) n'a, pour le moment, aucun statut théorique : il ne sert qu'à montrer l'absence de tout nom-tête explicite dans le DP. En fait, on verra qu'il y a de bonnes raisons de penser que s'il y a bien un tel " $\varnothing$ " dans les structures proprement elliptiques, comme en (7), où le nom-tête absent est donné par le co-texte immédiat (et est donc sémantiquement récupérable) - et donc aussi dans une interprétation contextualisée, et par suite spécifique, de (6) -, il n'y a, au contraire, rien entre -(e)n- et - $a$ dans la structure syntaxique sous-jacente à l'interprétation générique de cet exemple, paraphrasable alors par 'quiconque commettra une faute...'.

(7) Patxiren liburuak eta [[zuk idatzi dituzu.n].Ø].ak ${ }^{9}$

Patxi-GEN livre-PL(ABS) et toi-ERG écrit AUX- $n$-Ø-PL(ABS)

'Les livres de Patxi, et ceux que tu as écrits' [lit. ...et les que tu les as écrits]

L'autre remarque, également anodine en apparence, se révélera tout aussi significative. Comme on peut s'y attendre dans la mesure même où les RSL sont closes par une marque de nombre (suivie éventuellement d'une marque de cas) et doivent donc être considérées comme des expressions nominales ou DP à part entière ${ }^{10}$, et non d'éventuels purs $\mathrm{CP}$ - comme le sont peutêtre les RL au sens strict illustrées en $(3)^{11}-$, l'éventuel suffixe casuel qui suit cette marque de nombre est normalement déterminé par la fonction que joue le DP dans la matrice qui le contient, et non par la fonction que joue la position relativisée à l'intérieur même de la relative. De ce point de vue, le paradigme standard est fourni d'un côté par l'équivalent sans dislocation de (1) donné en (8), où le cas qui correspond à la position relativisée dans la RSL, i.e. l'ergatif indiquant le sujet d'un verbe transitif, est bloqué : c'est en effet l'absolutif, qui correspond à la fonction de cette RSL dans la matrice (celle d'un DP sujet d'un verbe intransitif "inaccusatif" -

\footnotetext{
${ }^{8} \mathrm{Si}$ l'on tient compte de l'ordre des mots illustré en (5d), le zéro pourrait tout aussi bien figurer à gauche, cf. la fin de 3.3 .

${ }^{9}$ Exemple simplifié (mais avec indication structurale par crochets et zéro ajoutés) tiré d'Euskaltzaindia (1999, p. 206).

Rappelons par ailleurs que, dans les dialectes orientaux comme dans la langue normalisée, le suffixe - $a k$ est ambigu entre l'ergatif singulier (segmentable en $-a-k$ ) et l'absolutif pluriel irrégulier non-segmentable, mais que cette séquence, dans les dialectes occidentaux parlés (et écrits jusqu'au milieu du $20 \mathrm{e}$ siècle), peut aussi avoir la valeur d'ergatif pluriel (elle équivaut alors au basque oriental et standard $-e-k$, où $-e$ - est la marque non-terminale du pluriel, et $-k$ le suffixe ergatif), si bien qu'un grand nombre d'exemples sont formellement ambigus, et ne peuvent donc pas être comptés parmi les tours non-standard examinés ici.

${ }^{10}$ Beñat Oyharçabal (communication personnelle) m'a fait remarquer qu'il existe des arguments poussant à distinguer entre $\mathrm{DP}$ (la projection d'un $\mathrm{D}^{\circ}$ ou Déterminant introduisant un $\mathrm{NP}$ ), et $\mathrm{KP}$, projection d'une tête fonctionnelle $\mathrm{K}^{\circ}$ réalisée par un suffixe casuel ou certaines postpositions, qui prendrait le DP comme complément. Je n'introduis pas cette distinction ici, dans la mesure où les arguments en question reposent essentiellement sur les propriétés combinatoires des pronoms sous coordination, question qui ne me paraît pas cruciale ici (voir cependant 4.5 sur ce sujet). Par ailleurs, il faudrait aussi s'interroger sur l'éventuelle nécessité de recourir à une tête fonctionnelle éventuelle Nombre ${ }^{\circ}$ ou Num ${ }^{\circ}$, et à sa projection NumP (pour Number Phrase), en particulier pour des cas comme ceux décrits en 3.2, ou les ex. (A5), (A44) (C25) de l'Appendice.

11 Je ne vois pas d'argument convaincant en faveur la thèse selon laquelle, lorsqu'il y a dislocation à gauche, ce qui est le cas le plus usuel, les RL strictes du basque seraient autre chose que de simples CP, directement interprétables comme des propriétés liant une variable de propriété dans la traduction sémantique du pronom de reprise ou corrélat - cf. Rebuschi (1999). Mais la question est évidemment plus complexe lorsqu'elles ne sont pas disloquées : voir Rebuschi (sous presse) pour quelques exemples manifestant l'absence de congruence casuelle dans de tels cas.
} 
en fait, passif ici), qui est sélectionné — et d'un autre côté par (9), qui présente la structure inverse : l'ergatif est maintenant obligatoire sur le DP, car le prédicat dont cette entité est le sujet est un groupe verbal transitif, ce qui bloque l'absolutif de (9b) qui correspond à la fonction relativisée interne à la $\mathrm{RSL}$.

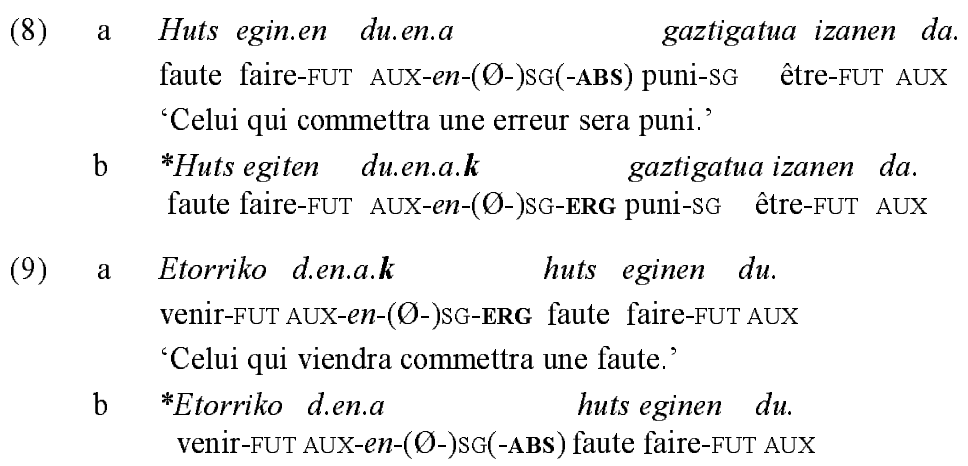

2.4. Nous sommes maintenant en mesure de présenter explicitement le phénomène qui nous concerne au premier chef. Rappelons d'abord qu'en basque comme ailleurs, il est possible de topicaliser un argument (ou un circonstant) en l'isolant prosodiquement de la proposition à laquelle il appartient sémantiquement - on parle alors plus techniquement de dislocation. ${ }^{12}$ Quant on disloque ainsi une relative libre, il ne se passe pas grand-chose en basque standard, si ce n'est la présence optionnelle d'un pronom de reprise ${ }^{13}$ à droite de l'élément disloqué, comme dans les paraphrases suivantes des exemples précédents:

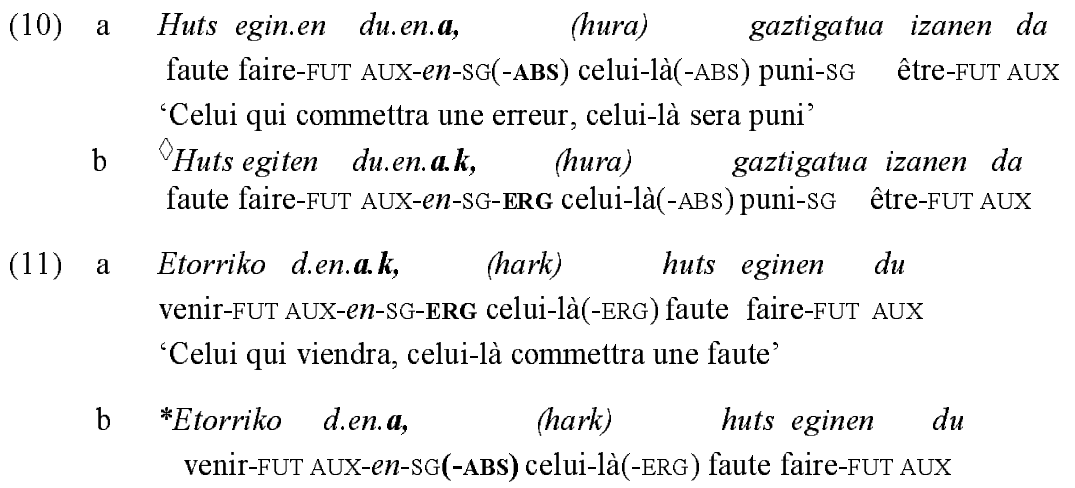

On constate que le cas de la RSL disloquée doit être identique au cas du pronom de reprise en position argumentale - ou encore au cas qu'il manifesterait s'il était réalisé, au cas où il ne le serait pas. Il s'ensuit que (10b) et (11b) sont en principe aussi agrammaticales que les variantes sans dislocation que sont $(8 \mathrm{~b})$ et $(9 b)$.

Et c'est ici que se produit la surprise : en fait, de très nombreux textes attestent l'existence, récurrente à travers les siècles et les dialectes, de structures de type (10b) - ce qui explique que j'aie choisi d'employer un autre symbole que l'astérisque pour l'introduire, ce dernier symbole signalant non seulement des phrases agrammaticales, mais encore la reconstruction de formes inattestées.

\footnotetext{
12 Comme les pronoms ne sont pas obligatoirement réalisés en basque (v. la note suivante), la différence entre la dislocation, qui exige la reprise par un pronom en français, et la topicalisation au sens strict, qui correspond à un déplacement laissant une trace, i.e. une catégorie phoniquement vide, est moins visible dans cette langue qu'en français. Cependant, outre la pause, la dislocation se distingue aussi par le fait qu'elle n'est soumise à aucune contrainte de localité, ce qui correspond bien au phénomène étudié.

13 L'optionnalité n'est que superficielle : dans la perspective générative, c'est un élément syntaxiquement et sémantiquement actif, mais phoniquement non-réalisé, pro, qui occupe la place de hura en (10) et et de sa variante ergative hark en (11). Voir aussi Zwart (1998, p. 380) pour l'hypothèse que même dans une langue non-pro-drop comme le néerlandais, les adverbes placés en position périphérique gauche sont "standardly resumed by an empty D-word".
} 
Le fait que les constructions comme $(1 \mathrm{~b}) /(10 \mathrm{~b})$ soient considérées comme fautives ne change rien à l'affaire; il faut en effet expliquer au moins trois choses, à savoir :

(i) l'asymétrie entre les exemples de ce type, dont on vient de dire qu'ils sont bien attestés (voir l'Appendice pour une bonne centaine d'exemples réels) et les exemples de type (1lb), qui sont par contre quasiment absents des textes ${ }^{14}$, enfin,

(ii) pourquoi ce phénomène ne se produit généralement que lorsque la RSL est disloquée, et

(iii) pourquoi il n'est pas attesté dans les cas d'ellipse lexicale sémantiquement récupérable, mais seulement autrement, cf. (7).

Après une présentation générale du cadre syntaxique adopté dans la section 3 , je proposerai une solution précise dans les trois suivantes, répondant au fur et à mesure aux questions posées à l'instant.

\section{Le cadre et les enjeux syntaxiques}

3.1. Je ne vais pas chercher à discuter ici des diverses hypothèses qui ont été émises concernant la structure interne des relatives libres au sens strict : depuis Bresnan \& Grimshaw (1978), Groos \& van Riemsdijk (1979) et Hirschbühler \& Rivero (1983), la discussion a été essentiellement consacrée à la question de savoir si l'élément $w h$ - visible est interne à la relative, et donc en Spéc,CP (COMP à l'époque), ou bien s'il joue le rôle d'un antécédent externe à la relative, à la manière d'une "tête" lexicale) ${ }^{15}$; or ces questions concernent les structures de type (2) ou (3), en particulier quand elles sont en position argumentale, plutôt que les RSL, qui manifestent des propriétés opposées : présence d'un Dét. explicite, et absence de pronom relatif visible. L'hypothèse, que je défends par ailleurs, d'une interprétation des RL disloquées comme étant de purs $\mathrm{CP}$ (voir la note 11) est donc non-pertinente ici : nous avons affaire à des expressions clairement nominales du point de vue de leur périphérie droite, et de leur rapport au reste de la phrase dans laquelle elles apparaissent.

De ce point de vue, deux questions formelles se posent :

(i) Doit-on adopter l'analyse (commune jusqu'à Barriers) des expressions nominales comme étant des projections de N, soit des NP, ou plutôt celle dite de l'hypothèse DP, qui prend le Dét. comme tête, et le $\mathrm{N}^{\prime}$ de l'ancienne configuration comme un NP complément de ce $\mathrm{D}^{\circ}$ ?

(ii) Doit-on considérer les relatives restrictives, dont les RSL semblent bien être un sous-type (le premier domaine de restriction du quantificateur étant simplement non identifié, cf. le " $\varnothing$ " de (6)) comme adjointes à la projection maximale (NP ou DP) ou bien comme étant adjointes à une projection intermédiaire (respectivement N' ou NP) ? Noter d'ailleurs que si l'on adopte l'hypothèse DP, une nouvelle possibilité se présente, qui consiste à considérer que le CP relatif est dans la position du complément structural de $\mathrm{D}^{\circ}$ : on y reviendra en 3.3 .

3.2. L'hypothèse DP n'est plus vraiment une hypothèse aujourd'hui, mais une analyse standard, au moins pour les langues qui ont des déterminants lexicalisés. En ce qui concerne le basque, il existe divers arguments qui la corroborent, si bien qu'elle a été adoptée sans discussion dans les sections précédentes. Mais il faut en dire quelques mots ici.

\footnotetext{
14 Voir cependant les ex. (B10,14,18-20) de l'Appendice, tous issus de deux dialectes voisins, le baztanais et l'aezcoan. Cette asymétrie est telle qu'elle permet d'exclure sans discussion l'hypothèse d'une rupture de construction, l'auteur pensant d'abord employer un verbe transitif, puis optant pour un intransitif / inaccusatif: si c'était le cas, les exemples de type (11b) devraient être approximativement aussi nombreux que les autres, ce qui est parfaitement inexact. Noter aussi l'existence de corrections à des ms., qui ou bien n'enlèvent pas la marque non-standard d'ergatif (cf. la note 4 de l'Appendice, voire l'ajoute (comme signalé dans la note 63 de cet Appendice; voir aussi le commentaire qui y précède l'ex. (A12)). L'hypothèse de simples coquilles pour les textes imprimés est également à écarter en fonction de la même asymétrie statistique.

15 Pour une mise au point récente, cf. van Riemsdijk (2000).
} 
Tout d'abord, si la notion de paramètre des têtes a un sens, il est clair qu'un élément comme le - $a$ final des expressions nominales a plus de raisons d'être considéré comme une tête prenant son complément structural sur sa gauche que comme un spécificateur situé à droite du NP du début des années 80 .

Plus important, adopter l'hypothèse DP, c'est prédire qu'il existe une position de spécificateur de ce DP - une position que l'ancien NP ne permettait pas d'envisager, puisque, comme on vient de le rappeler, son spécificateur était le déterminant lui-même. Or, comme le montre en particulier Artiagoitia (1998), cette position est fréquemment utilisée en basque (voir aussi Eguzkitza (1995)). Ainsi, un pronom personnel peut occuper cette position, comme en (12), où les patenthèses rappellent qu'un pro est également disponible. De plus, spécifiquement en biscayen, un démonstratif peut aussi l'occuper, dupliquant ainsi la tête, cf. (13b) vs. (13a), autres dialectes.

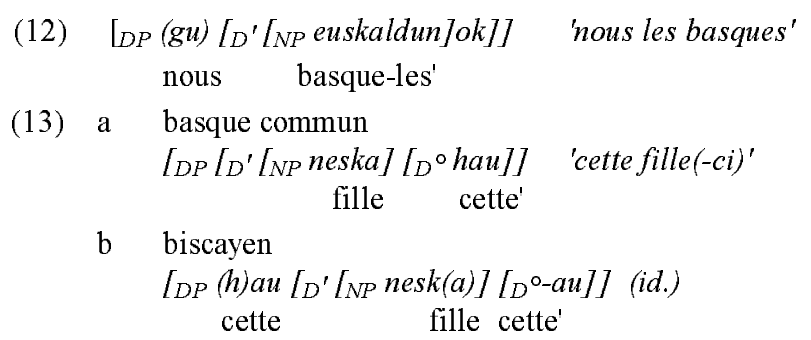

On peut donc tenir le DP comme acquis pour le basque. Cela dit, l'ex. (12) est particulièrement intéressant en ce qu'il fait ressortir l'existence visible d'un accord spécificateurtête en personne et en nombre dans les dialectes du Sud. En effet, l'article -ok est [-distant] ou [III], en ce sens qu'il n'apparaît qu'avec des pronoms spécificateurs de le ou 2e p. du pl., mais jamais de la $3 \mathrm{e}$ p. Qui plus est, cet accord peut n'être pas visible dans la morpho-syntaxe interne des expressions nominales, comme dans les dialectes tels qu'ils sont écrits ou parlés aujourd'hui au Nord, mais il revient tout de même à la surface dans ces dialectes dans la mesure où la forme verbale fléchie doit s'accorder en personne et nombre avec ce pronom - c'est-à-dire avec le DP en tant qu'il hérite ces traits de sa Tête $\mathrm{D}^{\circ}$, traits qu'elle n'a elle-même pu obtenir que grâce à l'accord Spéc-Tête :

(14) a Nord

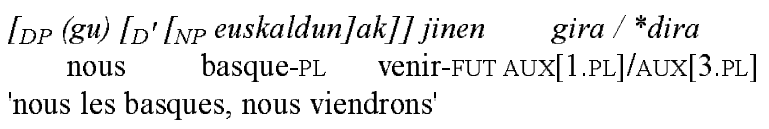

b Sud

$$
\begin{aligned}
& {\left[D P ( g u ) \left[D^{\prime}[N P \text { euskaldun]ok]] etorriko gara/*dira }\right.\right.} \\
& \text { nous basque-PL[-III] venir-FUT AUX[1.PL]/AUX[3.PL] }
\end{aligned}
$$

Quant à (13b), il va de soi que seul un démonstratif de distance identique (de distance I, II, ou III) peut être dupliqué en biscayen, ce qui est une forme encore plus forte d'accord.

3.3. La seconde question posée en 3.1 ci-dessus est apparemment plus ouverte que la précédente, parce que l'on sait, depuis Bach \& Cooper (1978), qu'il est possible de calculer compositionnellement l'interprétation intersective des relatives restrictives à partir d'une représentation dans laquelle la relative est adjointe à la projection maximale de l'expression nominale, et non pas à une projection intermédiaire. Cependant, si l'on n'a pas de bons arguments syntaxiques pour défendre cette analyse structurale, dans la mesure même où l'adjonction à DP est généralement considérée comme le mode de rattachement des relatives appositives, qui sont clairement en dehors du champ de quantification du déterminant, je pense préférable (contre van Riemsdijk (2000) par ex.) d'adopter cette représentation standard, qui permet au déterminant de quantifier l'ensemble obtenu par l'intersection de l'ensemble fourni par le NP et de celui fourni par la relative ${ }^{16}$.

\footnotetext{
16 Voir déjà la discussion dans Chomsky (1974), qui se résout aisément aujourd'hui avec l'hypothèse DP - qu'il a lui-même acceptée depuis une dizaine d'années.
} 
En d'autres termes, en l'absence d'arguments allant dans le sens contraire, on analysera les relatives restrictives basques comme adjointes au NP interne au DP, comme dans (15), qui reprend (5c) - plutôt que comme (16) :

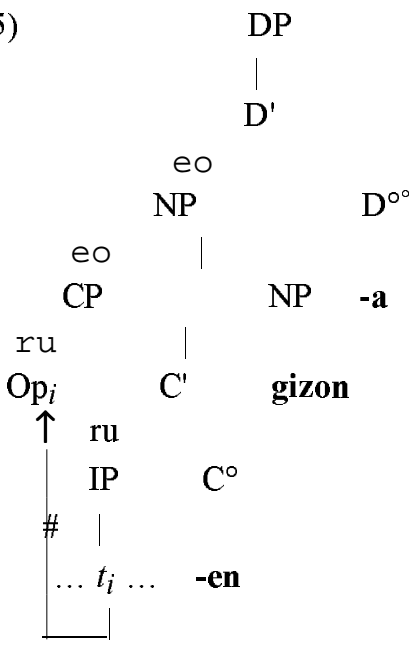

(16)

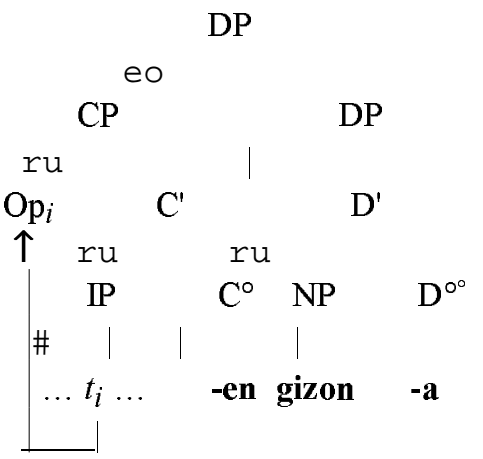

Ajoutons que, du point de vue empirique, (16) est une représentation qui, crucialement, ne permet pas de construire l'ordre alternatif NP-CP illustré en $(5 \mathrm{~d})$; en effet, à moins de déplacer de manière ad hoc le NP inférieur gizon vers la position, non activée ci-dessus, de Spécificateur $\mathrm{du}$ DP du haut, on ne peut en rendre compte ; avec (15) par contre, il suffit d'adjoindre le CP relatif à droite du NP pour obtenir le résultat voulu.

3.4. Considérons maintenant la partie exploitable des hypothèses de Kayne (1994), à savoir, l'idée qu'un $\mathrm{CP}$ pourrait bien se trouver dans la position du complément de $\mathrm{D}^{\circ}$, au lieu d'être adjoint au NP de $(15)^{17}$. Cette hypothèse permet de construire la représentation (17) :

\footnotetext{
${ }^{17}$ Par contre, je n'adopte évidemment ni l'idée qu'il n'y a jamais de NP auquel une relative restrictive serait adjointe, ni celle, corrélée, que le mouvement est celui de l'antécédent, ni non plus, enfin, celle qui revient à dire que les têtes précèdent toujours leur complément - pour retrouver l'ordre des mots de $(5 \mathrm{c})$, il faudrait en effet considérer que tout ce qui précède le $\mathrm{D}^{\circ}$ (le CP lui-même selon Kayne), a été déplacé en Spéc,DP; de plus, ce CP aurait subi de multiples déplacements internes, car le C' est passé à gauche de gizon, qui par hypothèse serait dans le $\mathrm{Spec}, \mathrm{CP}$ : il faudrait donc en réalité admettre une itération de $\mathrm{CP}$, permettant la montée ultérieure dudit $C^{\prime}$ dans le Spéc. du segment supérieur de CP, etc.
} 
(17)

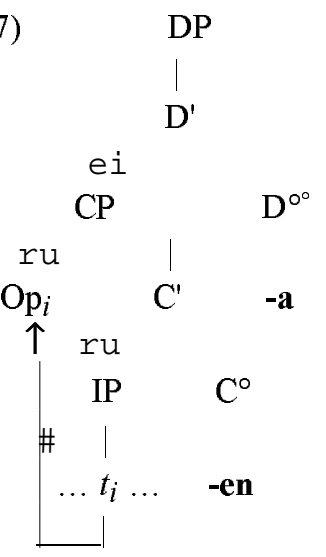

$\mathrm{Si}$, comme on va le rappeler plus en détails infra, tout opérateur ou pronom relatif est sémantiquement un opérateur d'abstraction, transformant une proposition en prédicat ou fonction caractéristique d'un ensemble, prendre un $\mathrm{CP}$ comme complément d'un $\mathrm{D}^{\circ}$ n'est, clairement, pas un problème sémantique. La question qui se pose est plutôt de savoir s'il existe des arguments concrets permettant de choisir entre (17) et (18) - la variante à "tête lexicale " vide (en fait, à NP vide) de (15) :

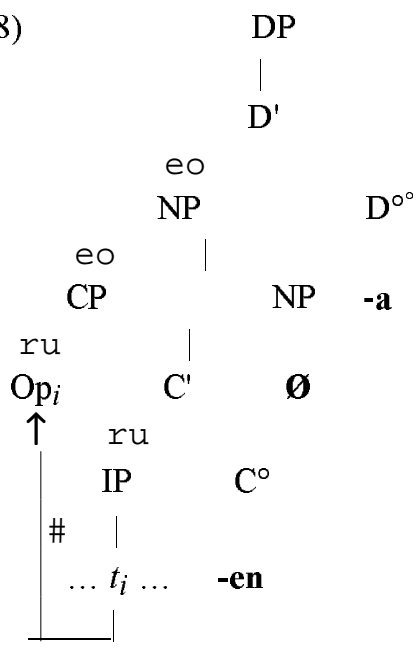

\section{Vers une solution unitaire}

4.1. Ma réponse est positive, mais, avant d'en exposer les arguments, je préfère exposer dès maintenant mon hypothèse, qui est la suivante : en ce qui concerne les DP à tête lexicale explicite et ou elliptée, mais contenant une relative restrictive, leur structure est respectivement celle de (15) et (18), qui est la plus simple du point de vue et de la syntaxe, et de linterprétation sémantique, et ne demande donc aucune argumentation particulière. Par contre, pour ce qui est des RSL non elliptiques, leur structure syntaxique serait celle donnée par (17), qu'elle soit « générée dans la base », pour reprendre une expression passée de mode, mais reformulable en termes de merging, ou le fruit d'une réanalyse de (18) à partir du moment où certaines conditions de transparence du NP «nul» y seraient vérifiées. Pour traiter de la percolation de la marque d'ergatif vers le haut, l'idée est alors la suivante : l'opérateur phoniquement vide se déplacerait de 
Spéc, CP vers Spéc,DP ${ }^{18}$, cf. (19), et de là, pourrait transmettre, sous certaines conditions, son trait de cas à la tête $\mathrm{D}^{\circ}$, la configuration tête-spécificateur permettant précisément ce genre d'accord.

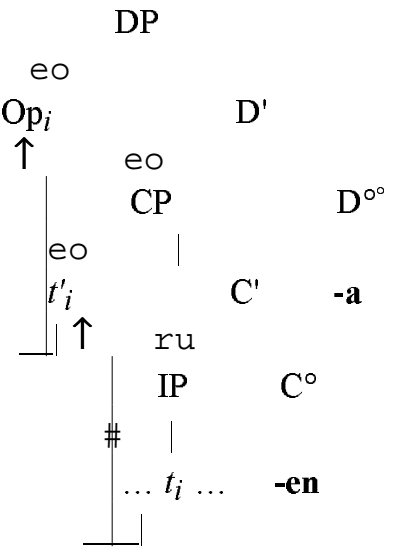

4.2. Nous avons donc un problème descriptif à résoudre, celui de l'analyse précise des RSL non-standard (1b) / (10b) (et, rappelons-le, de la quasi-absence d'exemples inversés, cp. (11b)) d'un côté, et celui, plus théorique, du choix entre (17) et (18), ou de l'éventuelle justification de l'adoption de ces deux structures en grammaire basque, de l'autre.

La question descriptive, on l'a dit et redit, est fondamentalement celle-ci : comment la marque casuelle correspondant à la position jouée par la position relativisée peut-elle apparaître (linéairement) sur la tête du DP contenant le CP relatif ? Il n'y a évidemment pas de processus d'assignation casuelle à envisager, puisque si assignation casuelle il y a, elle doit se faire de l'extérieur, par une tête qui gouverne ou régit le DP entier. La seule solution offerte par la théorie des Principes et paramètres semble donc être la suivante : l'apparition du suffixe ergatif inattendu, «fautif», résulterait d'un accord entre tête et spécificateur, cette relation structurale étant l'une des plus fondamentales de cette théorie ${ }^{19}$. Or c'est seulement si la position de spécificateur de DP est disponible, i.e. potentiellement présente mais non occupée, qu'un élément venant de plus bas pourra s'y placer. Quel peut alors être cet élément ? Le candidat le plus naturel ne peut qu'être l'opérateur ou relatif vide - d'une part parce qu'étant non-réalisé phoniquement, son mouvement est invisible du côté de la face phonétique de la phrase, et d'autre part parce qu'il est bien, lui, doté, sous forme de trait, du cas précis correspondant à la position relativisée dans la relative (on verra une justification sémantique de ce déplacement au § 6). Sous accord spécificateur-tête, ce trait de cas pourrait donc être transmis au Dét., la tête du DP : on a vu indépendamment que le basque pratiquait l'accord Spéc,tête dans ses DP - cf. les ex. (12) et (14), et la discussion qui les suit $^{20}$.

18 Jen reste donc, à l'adoption des projections CP et DP près, à une analyse très proche de celle suggérée par Oyharçabal (1988). Cet auteur a ensuite proposé, cf. Oyharçabal (1989), une solution alternative au déplacement de l'opérateur, selon laquelle ce dernier serait généré dans la base en Spec, CP, et lierait un pro résomptif. Le choix entre ces deux approches est indifférent à mon propos, car il importe peu, en fait, de savoir si l'Op. dans Spéc,CP en (15), (17) et (18), y est généré directement, ou s'y trouve suite à une application de Move $\alpha$ : c'est le mouvement suivant, vers Spéc,DP, qui est crucial pour moi ici.

19 Elle est même devenue pratiquement la seule opératoire dans le Programme minimaliste, et correspond exactement à la notion de propagation des «traits-de-tête» ou head features en PSG.

${ }^{20}$ En termes minimalistes, donc vérificationnistes, on pourrait dire - ce qui revient dans les faits au même - que la marque casuelle ergative sur le $\mathrm{D}^{\circ}$, si elle ne peut se vérifier de manière usuelle, i.e. par rapport au reste de la phrase, peut se vérifier de manière interne au DP, ce qui ne peut se faire que si l'Opérateur abstrait monte de Spéc,CP en Spéc,DP : l'intuition d'un mouvement « altruiste» semble ici beaucoup plus vraisemblable que celle d'un mouvement «égoïste», car on ne voit guère ce qui, dans ces termes, pourrait expliquer la montée de l'Opérateur $w h$-de manière non ad hoc et non-circulaire. 
La raison interne de cette montée de l'opérateur en Spec,DP m'est inconnue, mais je ne crois pas que les spécialistes qui ont décrit le mouvement "trop long" des éléments $W h$ - dans certains dialectes non-standard de l'allemand aient pu avancer de véritable explication syntaxique. Dans le cas qui nous préoccupe, par contre, on a au moins une explication fonctionnelle, externe, et un pendant sémantique, ce qui est déjà un début.

4.3. Cette motivation externe, fonctionnelle, peut se présenter comme suit. Il faut d'abord se souvenir du fait déjà cité que, "normalement", les RSL non-standard ne portent extérieurement le cas ergatif de la position relativisée que lorsqu'elles sont disloquées à gauche ${ }^{21}$. Cela semble indiquer qu'il peut y avoir parfois absence de transmission du cas porté par le pronom argument (ou circonstant) de la matrice vers le DP (la RSL) disloqué qui lui est coindexé, phénomène qui n'est pas rare à travers les langues (cf. van Riemsdijk 1997).

Cette hypothèse est clairement corroborée par les seuls exemples de RSL non-standard trouvés chez Uriarte, biscayen du $19^{\mathrm{e}}$ siècle qui a traduit de nombreux textes soit en biscayen, soit (surtout) en guipuzcoan. Il s'agit de cas où, pour une raison ou une autre, il n'y a pas de reprise de la RSL disloquée dans l'apodose. En voici un exemple, tiré de sa Bible manuscrite en guipuzcoan :

(20) Proverbes, 28,24 (Uriarte \pm 1860 , Salomonen ...)

$\begin{array}{lccc}\text { Bere aitari eta bere amari } & \text { zerbait } & \text { kentzen diona }{ }^{\diamond} \text {, } \\ \text { son père-DAT et sa } & \text { mère-DAT quelque chose enlevant AUX-en-SG-ERG } \\ \text { eta esaten duena }{ }^{\diamond} \boldsymbol{k} & \text { ez dala au bekatu, gaiztakeria au da }\end{array}$

eta esaten duena $\boldsymbol{k}_{\boldsymbol{k}} \quad$ ez dala au bekatu, gaiztakeria au da
et disant AUX-en-SG-ERG NEG AUX-C ${ }^{\circ}$ ceci péché méfait celui-ci est

gizona iltzen duenaren gaitzakeriaren antzekoa.

homme tuant AUX-en-SG-GEN méfait-GEN comparable

Lit. 'le-ERG [=celui] qui prend quelque chose à son père ou à sa mère, et qui ${ }^{22}$ dit que ce n'est pas un péché, ce méfait est comparable au méfait de celui qui tue un être humain.'

Comme on le voit, la RSL disloquée n'est pas reprise par un pronom, même vide, puisque gaiztakeria au 'cette méchanceté' réanalyse conceptuellement et lexicalement le contenu du DP disloqué, qui dénote un individu, en un fait. Si l'on est en droit de considérer que la période entière est mal construite, en raison même de la non-reprise de la RSL, cet accident (car Uriarte est un auteur généralement très soigneux ${ }^{23}$ ) illustre ce qui vient d'être dit : il ne saurait y avoir de transmission casuelle à partir d'une position argumentale occupée par un pronom de reprise, puisqu'il n'y a, tout simplement, pas de reprise ${ }^{24}$.

4.4. Admettons donc que les RSL disloquées du basque ne reçoivent pas toujours de cas à partir du pronom résomptif qu'elles lient. On est alors en droit de se demander pourquoi cette langue n'a pas recours à la stratégie bien connue d'emploi du cas par défaut. Or il se trouve qu'en basque, il n'est pas facile de dire quel est le cas non-marqué, et même s'il y en a un qui serait clairement tel. En effet, c'est l'absolutif qui est, morphologiquement, le cas nul ; mais, par

21 Voir l'Appendice pour des exemples de RSL à droite qui occupent en fait une position également nonargumentale.

22 On va revenir sur la duplication $\mathrm{du} \mathrm{D}^{\circ}$ (et de la marque casuelle éventuelle) en 6.2 .

23 Sauf erreur, je n'ai relevé aucune RSL non-standard dans ses deux trad. guipuzcoanes de Matthieu, , et une seule, dans un cas structuralement identique à celui de (20), dans sa trad. biscayenne (cf. l'Appendice, ex. (A44)).

24 Il me faut signaler ici qu'au printemps 1999, au cours de discussions concernant les "vraies " relatives libres avec Jean Haritschelhar, Président de l'Académie basque et natif de St.-Etienne de Baïgorry (Basse-Navarre), ce dernier employait très systématiquement des DP à marque ergative externe lorsque la RSL, considérée comme variante de la RL stricte, était employée en isolation, plutôt que comme argument d'un verbe ; il opposait ainsi des formes comme (1a), reliées à un pro ou un pronom explicite, et donc reliées indirectement à un prédicat, à des formes comme (i), ci-dessous, qui ne lui venaient, j'y insiste, qu'en l'absence de tout contexte:

(i) Huts egiten du.en.Ø. $\mathrm{a}_{\mathbf{k}}$ erreur faire-IMP AUX-en-Ø-SG-ERG 
ailleurs, c'est l'ergatif qui, dans les constructions transitives ou intransitives prétendûment «inergatives » est le cas du sujet : l'absolutif ne peut donc pas servir syntaxiquement de cas par défaut, tandis que l'ergatif ne peut pas servir morphologiquement de cas par défaut. Il s'ensuit que la première stratégie n'est pas disponible, d'où le recours au mouvement de l'opérateur, qui va transmettre son cas à la tête $\mathrm{D}^{\circ 25}$.

On se trouve donc confronté à une forme de «bricolage », ou de stratégie de dernier recours en raison de l'indisponibilité du soi-disant dernier recours généralement employé à travers les langues : si, dans ces conditions, il n'est pas étonnant que ces constructions aient un statut marginal, on va cependant voir en 6 qu'elles sont aussi aussi le réflexe morpho-syntaxique d'une opération sémantique indépendamment justifiée 26

En résumé, si l'on admet que le filtre casuel, qui exige que tout DP soit marqué d'un cas, est, d'une manière ou d'une autre, opératoire, deux stratégies sont disponibles pour fournir un cas à un DP disloqué27: ou bien il peut être doté intrinsèquement du cas non-marqué dans la langue (ou le recevoir par défaut), ou bien il peut recevoir un cas interne, par montée de l'opérateur en Spéc,DP puis accord entre ce spécificateur et la tête, comme je le propose ici.

4.5. Le rôle de la morphologie (concrète) est probablement également à invoquer doublement pour rendre compte de l'asymétrie entre (10b) et (1lb). En effet, l'ergatif du pronom de reprise dans la matrice étant réalisé concrètement, par un segment audible, il semble que la transmission du cas de ce pronom au DP disloqué se fasse beaucoup mieux. Et il ne s'agit sûrement pas ici d'une question de «performance» en ligne, puisque le suffixe ergatif visible sur le pronom suit la RSL, mais d'une question plus complexe, probablement liée au fait que l'absence de tout segment matérialisant l'absolutif correspond peut-être à une distinction morphosyntaxique entre DP pur et KP, question délicate qui mériterait un approfondissement (cf. la note 10). Par ailleurs, la plupart des cas bien connus d'assignation parasitaire de cas reviennent généralement à ajouter un segment, et pas à en retrancher un ${ }^{28}$.

Un argument indépendant en faveur de cette thèse est d'ailleurs fourni en creux par l'extrême rareté de l'apparition du suffixe ergatif correspondant au cas de la position relativisée dans la RSL lorsque le DP disloqué, i.e. la RSL, correspond à un argument au cas datif - marqué par un segment visible - dans la principale (mais l'Appendice nous indique aussi qu'une infime fraction de RSL nst. présente le «remplacement» d'un affixe visible (ergatif) le zéro absolutif, exemples qui semblent indiquer que «l'exception confirme la règle»).

4.6. On vient de voir par quels mécanismes syntaxiques (montée de l'opérateur vide puis accord spécificateur-tête) le cas interne de la RSL pouvait se trouver réalisé sur $\mathrm{D}^{\circ}$, la tête fonctionnelle placée le plus haut dans cette RSL. Il reste donc à montrer qu'il existe des justifications indépendantes à cette transformation. Ces justifications, vers lesquelles on va se tourner maintenant, sont autant d'ordre sémantique que syntaxique. On commencera par examiner en 5 l'opposition entre l'analyse à NP vide et l'analyse à la Kayne (cf. (17) et (18)) en

\footnotetext{
25 Voir, dans l'Appendice, la discussion qui précède les ex. (A13-16), qui semblent, de prime abord, exhiber un datif comme cas par défaut : en fait, une tout autre dimension est en jeu, qui dépasse largement le cadre de la grammaire de la phrase.

${ }^{26} \mathrm{Il}$ n'y a pas forcément contradiction : si la transformation syntaxique sous-jacente à l'opération en question a lieu avant "Spell-Out", elle aura un réflexe en PF ; mais si elle s'applique après, on est dans le cas du basque standard, qui ne permet pas qu'il apparaisse de marque visible de ladite opération : voir 7.3.

27 C'est ici qu'on voit bien la différence entre topicalisation (mouvement du DP) et dislocation : si un DP est déplacé, il va naturellement porter le cas reçu (ou "vérifié") en position de départ, avant mouvement. La situation est radicalement différente dans le cas des dislocations, puisqu'il n'y a pas de déplacement, l'élément disloqué étant " généré dans la base " en position non-argumentale ; cf. cependant Prince (1997) pour une vision un peu plus tempérée de la question.

28 Comme dans les formes hypercorrectes this is the girl who $\boldsymbol{m}$ I know loves Bill ou leurs équivalents hongrois, où c'est la trace intermédiaire du relatif, en Spéc,Comp de la relative, qui reçoit l'accusatif par marquage de cas exceptionnel ( $E C M)$; noter à cet égard que ce genre de construction est, que je sache, inattesté dans les langues où le nominatif est déjà marqué par un segment final, que l'accusatif en présente un autre ou n'ait pas de marque du tout.
} 
associant chacune à une interprétation sémantique différente, et en montrant qu'il y a une explication sémantique au blocage de la montée de l'opérateur vers Spéc,DP dans le cas de (18) Dans la section 6 enfin, on examinera un phénomène bien connu des bascologues, mais qui n'a jusqu'à présent (à ma connaissance du moins) fait l'objet d'aucune analyse formelle - je veux parler du fait que les RSL peuvent aussi fonctionner comme éléments adjoints à droite des DP (définis ou non), et pourtant s'interpréter comme des relatives restrictives : cette donnée incontournable du basque dans toutes ses variétés (standard ou non, et diachroniques aussi bien que dialectales) nous montrera que cette langue permet de manière très régulière et uniforme un changement de type des RSL, qui s'interprètent aussi bien comme des propriétés que comme des individus, le mécanisme responsable de ce type shifting généralisé étant en toute probabilité l'effet du même déplacement de l'opérateur vide que celui proposé jusqu'ici pour des raisons morpho-syntaxiques de Spéc, CP vers Spéc,DP.

\section{RSL elliptiques et non-elliptiques}

5.1. Le propre des lacunes elliptiques est qu'elles peuvent être remplacées par du matériel lexical. De ce point de vue, le zéro (Ø) de (7) pourrait être remplacé par liburu 'livre' sans aucun effet sémantique. Mais considérons maintenant (21):

\section{(21) Erortzen dena altxatu behar da.} tombant AUX-C ${ }^{\circ}$-SG lever devoir AUX

Cette phrase est susceptible de recevoir diverses traductions en français, de par le fait, entre autres, qu'il est impossible de savoir si l'on réfère à de l'humain (ou de l'animé), ou à du nonhumain (ou inanimé). Dans le second cas, on traduira par 'il faut relever ce qui est tombé', et, dans le premier, par 'il faut relever celui qui est tombé' ou encore, au moins dans les dialectes du Nord, par 'celui qui est tombé doit se relever'. En conséquence, il n'existe par le moindre indice de la présence d'un trait de catégorisation de type [ \pm humain] entre le $\mathrm{C}^{\circ}$ - (e)n et le $\mathrm{D}^{\circ}-a^{29}$ - en d'autres termes, le NP phoniquement vide est également sémantiquement vide.

Il est possible que, dans ces conditions, il soit en fait totalement absent, c'est-à-dire que, dans les notations superficielles enrichies des RSL d'interprétation non-elliptique, on n'ait rien, plutôt qu'un segment $\varnothing$, entre le $\mathrm{C}^{\circ}$-(e)n et la marque de nombre ou détermination - $a$ (comparer (6) supra) : nous avons donc ici un argument interne au basque pour choisir l'arborescence (17), plutôt que l'arborescence (18), dans le cas des RSL non-elliptiques. ${ }^{30}$

5.2. Mais cette hypothèse ne sera vraisemblable que si non seulement la montée de l'opérateur de Spéc,CP vers Spéc,DP est possible dans ce cas (cf. (19)), mais encore qu'elle est impossible dans le cas de (18), c'est-à-dire que si (22), où le [NP Ø] est porteur de traits lexicaux, n'est pas possible :

29 Bien entendu, le prédicat de la matrice peut orienter l'interlocuteur, comme dans les cas suivants, où notre connaissance du monde usuel nous pousse à interpréter la première RSL comme introduisant de l'humain (d'où la trad. par 'celui qui') et le second comme de l'inanimé (trad. 'ce qui'):

(i) Erortzen dena, hura lagundu behar da/dugu jeikitzen 'Celui/??ce qui tombe, nous devons l'aider à se relever'

(ii) Erortzen dena, hura txakurrarentzat (izaten) da. 'Ce/??celui qui tombe, c'est/??il est pour le chien'

$30 \mathrm{Il}$ y a deux façons de construire une telle structure : on peut ou bien associer (anglais merge) directement un $\mathrm{D}^{\circ}$ et un $\mathrm{CP}$ construit préalablement, ou bien réanalyser un arbre comme (18) en (17), mais à la condition expresse que le NP auquel est adjoint le $\mathrm{CP}$, i.e. le segment inférieur de NP en (18), ne possède aucune information sémantique (ce qui signifie évidemment que ni le $\mathrm{N}^{\circ}$ ni le spécificateur potentiel de ce NP ne doivent contenir de traits). Mais cette question me parait sans pertinence pour les problèmes traités ici. 


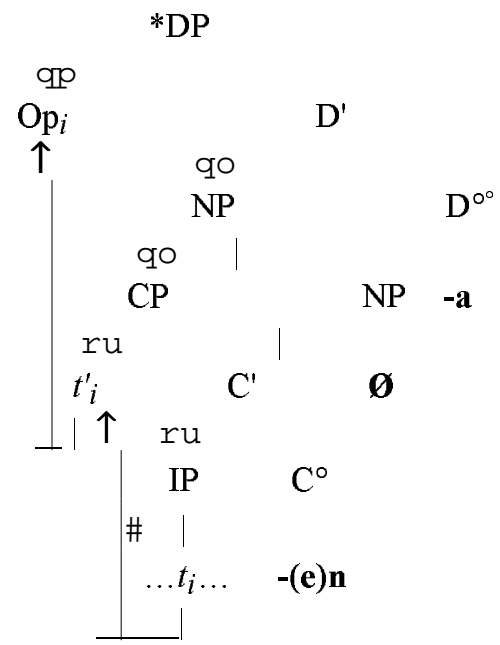

Dans ce cas, les corrélations respectives entre, d'une part, la structure (17), les RSL nonelliptiques, et la possibilité pour la marque d'ergatif interne d'apparaitre sur le $\mathrm{D}^{\circ}$, et, d'autre part, entre la structure (18), son interprétation elliptique (le NP phoniquement nul conservant ses traits sémantiques), et le blocage de la percolation vers le haut de la marque d'ergatif interne, seraient mieux assurée.

Qu'est-ce donc qui pourrait bloquer la montée de l'opérateur vide quand la relative est adjointe à un NP ? De par sa position même, la relative n'est pas exhaustivement dominée par NP, mais seulement par le segment NP supérieur; il s'ensuit qu'il ne saurait y avoir d'effet de barrière bloquant ce mouvement.

Il faut donc chercher une explication ailleurs que dans la syntaxe, et la sémantique en fournit une très simple. En effet, les relatives sont généralement considérées comme des prédicats ; par suite, on peut voir dans les pronoms relatifs des éléments qui sont tout simplement, sur le plan sémantique, des opérateurs- $\lambda$, i.e. des éléments qui transforment, par abstraction sur l'argument concerné, une proposition bien formée en une expression- $\lambda$ (le $\mathrm{CP}$ ), l'opérateur liant dans la proposition ouverte (l'IP) la variable qui correspond à la trace du relatif :

(23) $\left[\mathrm{C} " w h_{-i}\left[\mathrm{C}^{\prime} \mathrm{C}^{\circ}\left[\mathrm{I}^{\prime \prime} \ldots t_{i} \ldots\right]\right]\right] \Rightarrow \lambda x \cdot \mathrm{R}(x)^{31}$

L'interprétation intersective des relatives restrictives se calcule alors (presque ${ }^{32}$ ) compositionnellement, le NP tête du DP fournissant un premier prédicat, $\lambda x . \mathrm{P}(x)$ - ou toute variante alphabétique de cette formule, et la relative un second prédicat, cf. (23). La mise en facteur commun de l'opérateur $\lambda$ permet alors de coordonner les deux propositions ouvertes, le tout fournissant un prédicat complexe pour le NP global:

(24) a $\left[\mathrm{NP} \mathrm{NP}\left[\mathrm{C}^{\prime \prime} w h-i\left[\mathrm{C}^{\prime} \mathrm{C}^{\circ}\left[\mathrm{I}^{\prime \prime} \ldots t_{i} \ldots\right]\right]\right] \Rightarrow\right.$

b $\quad \lambda x \cdot \mathrm{P}(x) \& \lambda x \cdot \mathrm{R}(x) \quad \Rightarrow$

c $\lambda x[\mathrm{P}(x) \& \mathrm{R}(x)]$

Revenons maintenant à (17) et (18). Dans le cas de la première structure, s'il n'y a pas le second mouvement, indiqué en (19), le CP (comme propriété) se combine directement avec le déterminant (interprété comme un quantificateur, standard ou non), et l'on obtient un

\footnotetext{
31 Contre Dayal 1997, je pense donc qu'il vaut mieux considérer que le complémenteur lui-même est ininterprétable : il n'est là que comme tête permettant à un X" (le pronom relatif, ou le DP ou PP contenant un pronom relatif, en cas de pied-piping) de monter dans la position de spécificateur qui peut l'accueillir mais il est vrai qu'on pourrait tout aussi bien considérer que, par accord Spéc,tête, c'est le $\mathrm{C}^{\circ}$ qui joue le rôle de l'opérateur $\lambda$, le relatif devenant par contre invisible à l'interprétation sémantique.

32 On reste malgré tout obligé de stipuler que la structure d'adjonction [NP NP [CP]] ou [NP [CP] NP]] doit s'interpréter comme une coordination de prédicats, cf. (24b), où j'emploie le symbole " \&", bien qu'il ne s'agisse pas de la coordination de deux propositions, par souci de simplification (voir Link 1984 sur ce point).
} 
quantificateur généralisé, sans plus. Et, s'il y a mouvement, on admettra que l'opérateur vide ne déplace pas seulement son trait casuel (ou "formel") mais aussi ses propriétés sémantiques (qui sont évidemment non-lexicales) ${ }^{33}$. On obtient alors une situation dans laquelle le DP a comme tête un $\mathrm{D}^{\circ}$ interprétable comme un quantificateur, et comme spécificateur un opérateur- $\lambda$. Cette situation est clairement contradictoire, mais, toujours par accord entre le spécificateur et la tête, on peut admettre que la valeur intrinsèque du déterminant est effacée ou annulée, voire remplacée par celle d'opérateur- $\lambda$. Si l'on peut montrer que les RSL qui sont syntaxiquement de type $\left[\mathrm{CP}+\mathrm{D}^{\circ}\right]$ fonctionnent, ou en tout cas peuvent fonctionner, comme des prédicats (voir la section suivante là-dessus), la structure sera interprétable : ce prédicat liera en effet une variable de prédicat contenue dans le pronom de reprise, lui-même typiquement paraphrasable par quelque chose comme le $x$ qui a la propriété $R$.

Mais dans le cas de (18), la situation est différente. Voyons d'abord ce qui se passe si l'opérateur en Spéc, CP ne monte pas en Spéc,DP. On dispose alors de deux solutions distinctes. (i) Ou bien les traits sémantiques du NP élidé ont été conservés, et on se retrouve dans le cas de figure exposé en (23)-(24) - (ii) ou bien on adopte plutôt un traitement interprétatif/contextuel de l'ellipse ; dans ce dernier cas, au lieu d'être fourni par un NP (ou "nom commun") réalisé, le contenu lexical de $\mathrm{P}$ dans $(24 \mathrm{~b}, \mathrm{c})$ reste non-spécifié dans un premier temps, puis s'interprète pragmatiquement, et il n'y a rien à jouter à ce qui précède. Si, par contre, l'opérateur monte effectivement en Spéc,DP, comme l'indique la flèche de gauche de (22), la computation sémantique bloquera dès le moment où le NP ellipté, de contenu $\lambda \mathrm{x} . \boldsymbol{P}(\mathrm{x})$, devra se combiner avec l'interprétation du CP vidé de son opérateur relatif au niveau du segment NP supérieur : il n'existe en effet aucun moyen de coordonner deux objets sémantiques aussi différents qu'un prédicat et qu'une proposition ouverte. Il s'ensuit qu'une structure comme (22) est tout simplement ininterpétable, ce qui explique que les RSL non-standard interprétables elliptiquement ne soient pas attestées.

\section{Un corrélat sémantique fondamental}

Y a-t-il des indices de la montée de l'opérateur vide jusqu'en Spéc,DP qui soient indépendants des données morphologiques non-standard du basque ? C'est ce que l'on va établir dans cette dernière section.

6.1. Commençons par noter ou rappeler que les $S F R$ peuvent être syntaxiquement adjointes tout en étant sémantiquement restrictives, cf. (25), où le DP auquel la RSL est adjointe est défini, et (26), où il est indéfini (et dans la partie focale d'une proposition existentielle) :

(25) Etorriko da egun.a, [orok igurikatzen dugun.a]

venir-FUT AUX jour-SG tous-ERG attendant AUX- $n$-SG

'Le jour que nous attendons tous viendra'

(Oyharçabal 1987)

(26) Sardesen baditutzu izen bakar batzu,

à-Sardes $b a$-tu-les-as nom rare quelques

soinekoak zikindu ez dituzten.ak

vêtements sali NEG ils-les-ont- $\boldsymbol{n}$ - $\boldsymbol{a k}$

(Duvoisin 1865: Apoc 3,4)

'A Sardes, il y en a quelques-uns qui n'ont pas souillé leurs vêtements'

(Noter ici la différence par rapport aux appositives françaises ou anglaises : il s'agit, ou peut s'agir, de purs $\mathrm{CP}$ dans ces dernières langues, tandis que les RSL basques, y-compris quand elles sont adjointes, contiennent le Dét -a, et doivent donc s'analyser comme des DPs). Et l'on est bien sûr en droit de se demander comment cela peut se faire que ces DP puissent fonctionner comme des relatives restrictives.

\footnotetext{
33 De façon générale, on distingue entre le mouvement de catégories lexicales, qui en permet la reconstruction en forme logique, et celui d'items fonctionnels, qui, s'ils sont interprétables, comme précisément les opérateurs, ne sont pas interprétés dans leur position d'origine.
} 
6.2. La coordination de deux prédicats dans une RSL peut s'effectuer de trois manières différentes. La moins exotique consiste en l'utilisation de l'aux. suivi du complémenteur suffixal (e)n- à la fin de la structure coordonnée, comme en (27), où il est mis en facteur commun ${ }^{34}$. Il est également possible de faire l'ellipse de la seconde occurrence du verbe fléchi suffixé de -(e)n puis du Dét., comme en (28). Enfin, et c'est ce qui nous importe ici, il est possible de dupliquer cette forme fléchie suffixée, comme en (29) - ces trois ex. sont des extraits de Mt 5,19.

(27) (Biscayen : Kerexeta 1976)

[[IOnetariko agindurik txikiena ausi], eta [gizonai austen irakatsi]]

DEM ordre-de plus-petit-SG briser et aux-hommes à-briser enseigner

dagitse.n.a], Zeruetako Erreiñuan txikien.a izango da $\varnothing$;

AUX-en-SG du-ciel au-royaume le-plus-petit sera AUX (ABS)

[[[aginduok bete] ta [beteten irakatsi]] dagi.an.a]...

ordres-ces remplir et à-remplir enseigner AUX-en-SG

'Celui qui violera le plus petit de ces commandements, et enseignera aux hommes à les violer(, il)

sera le plus petit au Royaume des Cieux; celui qui suivra et enseignera à suivre ces

commandements ...'

(28) (basque unifié : $E H E G, 1980$ )

[[Betetzen du.en.a], berriz, [eta hala erakusten Ø]], haundia izango da... remplissant AUX-en-SG par-contre et ainsi enseigant grand sera AUX

'Celui qui [le] suivra, et l'enseignera, par contre, sera [tenu pour] grand'

(29) (Basque unifié : $E A B-1,1984)$

[[Betetzen duena] eta [betetzen irakasten du.en.a]], remplissant AUX-en-SG et à-remplir enseignera AUX-en-SG

ordea, haunditzat hartuko dute $\varnothing[\ldots]$

par-contre pour-grand prendront AUX (ABS)

Les RSL de (29), qui ne sont pas marquées pour l'ergatif, sont parfaitement standard mais, comme l'indique la forme verbale conjuguée (le $d$ - de $d u$ indique un objet direct de $3 \mathrm{e}$ p. du sg.), le pro (noté Ø) de reprise est un absolutif singulier, ce qui exclut que la coordination soit additive, et que l'on puisse interpréter chaque RSL comme dénotant un individu (maximal). Alex Grosu m'a cependant fait remarquer que la conjonction eta pourrait s'interpréter ici comme 'et/ou', laissant ainsi la possibilité d'un accord singulier un peu comme en français ${ }^{35}$. Or la conjonction baina 'mais', qui présuppose, au moins dans un contexte comme le suivant, un même référent, apparaît également avec ce tour, même avec un suffixe casuel qui n'est pas particulièrement léger :

(30) Ez izan beldurrik [[gorputza hiltzen dute.n.e.i],

NEG avoir peur corps tuant AUX-en-PL-DAT

baina [arima ezin hil dezaketenei]] (Mt 10,28 - Basque unifié : EHEG 1980)

mais âme NEG tuer AUX- $n$-PL-DAT

'N'ayez pas peur de ceux qui tuent le corps, et/mais ne peuvent tuer l'âme'

6.3. Une dernière propriété remarquable des RSL basques est qu'elles pouvaient être reprises jusqu'au $19^{\mathrm{e}}$ siècle, dans le dialecte labourdin, par un pronom très particulier, haina, dont j'ai montré ailleurs que le sens de base est celui d'un quantificateur universel associé à une variable

34 Cf. la parenthétisation, qui est de moi, comme dans les autres exemples.

35 Voir de ce point de vue les deux occurrences de eta dans l'ex. (20) supra, ainsi que la trad. de 'et' par ero[= edo 'ou] dans l'appendice, ex. (A3). 
de propriété (tout $x$ qui a la propriété $P$ ) - cf. Rebuschi (1998a) ${ }^{36}$. Ce qui compte ici, c'est son apport quantificationnel, qu'on peut illustrer par les deux faits suivants.

(a) Il n'apparaît jamais après une RSL disloquée dont le dét. est remplacé par un quantificateur universel comme oro ou guz(t)ia 'tout', comme en (31):

(31) Huts egiten duen guztia $^{37}$, hura/*haina gaztigatua izanen da. erreur faisant AUX-en tout lui / haina puni sera AUX 'Celui qui [lit. tout qui] commet une erreur, il sera puni.'

(b) Quand haina remplace un pronom démonstratif ordinaire dans l'apodose d'une phrase conditionnelle, aucune forme de quantification adverbiale ou «non-sélective » (par les équivalents basques de 'toujours', 'souvent', 'rarement', etc.) n'est attestée :

(32) Norbaitek huts egiten ba-du, hura/*haina maiz gaztigatua (izaten) da. quelqu'un-ERG erreur faisant si-AUX lui / haina souvent puni étant AUX 'Si quelqu'un commet une faute, il est souvent puni.'

Mais si haina est bien en fait à la fois un quantificateur universel et un pronom (tout $x$ qui $\boldsymbol{P}$ ), comment pourrait-il jamais incorporer le contenu sémantique d'une RSL non elliptique, si cette dernière se traduit par un individu pluriel maximal (ou bien est elle-même universellement quantifiée) ?

6.4. Ces propriétés grammaticales remarquables des RSL basques s'expliquent toutes très aisément si l'on admet qu'elles peuvent s'interpréter comme des prédicats. En effet, elles peuvent être syntaxiquement apposées, comme en (25) ou (26), si elles sont rendues sémantiquement licites par le fait même qu'elles lient une variable de prédicat associée optionnellement à la traduction du DP du bas (egun.a, izen bakar batzu) - cf. Bach et Cooper (1978). Les cas de coordination non-additive s'expliquent de la même manière : la coordination est en fait celle des propriétés, cf. (24b), si bien que la référence peut se faire au même individu pluriel (maximal). Enfin, s'il s'agit de propriétés, il n'y a plus de difficulté à leur reprise par le "pronom " haina tel que j'en ai indépendamment analysé la contribution sémantique.

Bien entendu, il n'est pas habituel du tout de voir la dénotation des expressions nominales passer si aisément du type $<\mathrm{e}>$ ou $<<\mathrm{e}, \mathrm{t}>, \mathrm{t}>$ en $<\mathrm{e}, \mathrm{t}>$, puisque c'est ordinairement lorsque ces expressions sont employées comme prédicats qu'elles subissent de changement de type (Partee 1987) : mais ce que la construction non-standard des RSL nous montre, c'est que l'opérateur relatif abstrait, en montant en Spéc,DP, peut transmettre son cas à la tête $\mathrm{D}^{\circ}$; dans ces conditions, on ne voit pas pourquoi le même mouvement ne pourrait pas avoir également un effet sémantique, qui reviendrait à surimposer au $\mathrm{D}^{\circ}$ sa valeur d'opérateur- $\lambda$, et donc à annuler la force quantificationnelle de ce déterminant : l'interprétation des RSL comme prédicats suit alors naturellement ${ }^{38}$.

\section{Résumé et conclusions}

\footnotetext{
36 C'est donc en gros un «pronom de type E », c'est-à-dire la matérialisation d'un type d'anaphore dont on connaissait le fonctionnement depuis Evans (1977), mais dont la lexicalisation spécifique dans les langues naturelles n'avait jamais été trouvée.

${ }^{37}$ L'usage de - $(e)$ n guz(t)ia au singulier pour renvoyer à de l'humain a disparu depuis près d'un siècle, le basque s'alignant ainsi sur les options des langues romanes environnantes : fr. tout ce qui, esp. todo lo que, vs. tous ceux qui, todos los que... Un ex. authentique est fourni par le passage suivant (avec renforcement de valeur purement stylistique [?] par la présence intemédiaire d'un démonstratif:

Mt 5,22 - Anon. B (guipuzcoan). Baña nik esaten dizutet ezen, bere anaiakin aserretzen dan hura guzia, hogendun izango da Ø juizioarena. 'Mais moi je vous dis que celui, quel qu'il soit, qui se fâche avec son frère, sera [jugé] coupable devant le tribunal.'

Un autre ex., avec marquage casuel non-standard, figure en (A17) dans l'Appendice.

${ }^{38}$ Le fait que des RSL standard (en $-a$ ou $-a k$, selon le nombre) puissent apparaitre sous la portée d'un opérateur existentiel dans les constructions du même nom va exactement dans le même sens - cf. aussi (26) supra.
} 
7.1. J'ai proposé de régler le problème morpho-syntaxique des relatives semi-libres nonstandard, qui consiste typiquement en l'apparition du cas ergatif qui correspond à la position relativisée interne à la subordonnée sur le DP qui contient celle-ci (DP qui serait normalement à l'absolutif), en suggérant que l'opérateur relatif (dont le cas est déterminé par la fonction de la variable qu'il lie dans la proposition relative) monte parfois de Spéc,CP en Spéc,DP, dans une configuration comme celle de (19), c'est à-dire dans une configuration généralement nonreconnue dans les travaux consacrés aux relatives libres ${ }^{39}$ (sauf dans Kayne (1994) évidemment, mais rien du reste de ses propositions n'est adopté ici), lorsque les conditions suivantes sont remplies

(i) la RSL n'est pas elliptique, et peut donc s'analyser (ou se réanalyser) comme un DP dont la tête $\mathrm{D}^{\circ}$ se combine avec un CP plutôt qu'un NP; et :

(ii) elle est disloquée à gauche, ce qui peut entraîner un blocage dans la transmission du cas à partir du pronom (plein ou vide) de reprise dans la proposition autonome à laquelle la RSL est adjointe.

Dans de telles circonstances, l'assignation du cas de l'opérateur relatif à la tête $\mathrm{D}^{\circ} \mathrm{du} \mathrm{DP}$, par accord entre spécificateur et tête, permet donc à la RSL de respecter le filtre casuel qui exige que tout DP soit muni d'un cas.

7.2. Du point de vue sémantique, les RSL basques, aussi bien standard que non-standard, ont un comportement apparemment étonnant, mais qui se ramène uniformément au fait qu'elles peuvent s'interpréter comme des prédicats, plutôt que comme des quantificateurs généralisés ou des entités (individus pluriels maximas). Un tel changement de type, rare à travers les langues, semble pouvoir s'expliquer par le même mouvement de l'opérateur relatif : interprété comme un opérateur- $\lambda$, une fois monté en Spéc,DP, et toujours par accord entre spécificateur et tête, il peut forcer le changement de type sémantique de la RSL, qui est syntaxiquement un DP, en une expression- $\lambda$ dont l'opérateur lie une variable d'argument, donc en un prédicat.

7.3. En conséquence, la différence essentielle entre les variétés standard et non-standard du basque du point de vue des RSL s'inscrit très naturellement dans la typologie descriptive de Chomsky $(1986)^{40}$, selon laquelle certaines différences entre diverses langues relèvent de la localisation d'une opération de mouvement soit avant la structure-s (ou "Spell-out"), soit entre cette dernière et la Forme Logique : dans tous les cas, ce mouvement a lieu (puisque le changement de type sémantique est général en basque), mais il n'y est morphophonologiquement visible que dans les variétés non-standard, dans lesquelles ce déplacement a lieu avant s-structure; dans les variétés conformes standard par contre, ce mouvement est phoniquement invisible, parce qu'il a lieu qu'après Spell-out ${ }^{41}$.

Centre de recherche en Typologie grammaticale (Tygre), Univ. Paris III \& UPRESA d'Etudes basques (Bayonne) $<$ rebuschi@ext.jussieu.fr $>$

\footnotetext{
39 Si l'on tient compte des travaux déjà anciens de Postal (1966), qui analyse les pronoms personnels comme des déterminants superficiellement «intransitifs », on voit par contre que des structures comme la suivante en sont peut-être une autre exemplification - mais il faudrait regarder de plus près si les hypothèses de Citko (sous presse) n'en rendent pas tout aussi bien compte :

(i) He who helps the blind deserves support. [Quirk et al. $1972: 862$ ] 'Celui qui aide les aveugles mérite d'être soutenu', lit. 'Il $\approx$ lui qui...'

$40 \mathrm{Il}$ y a sans doute aussi derrière ces probèmes tout un champ de données à analyser du point de vue de l'acquisition du basque comme langue première, mais je laisse aux spécialistes de ces questions le soin de s'y intéresser, ou pas...

41 On comprendra qu'avec les dernières péripéties concernant l'hypothèse de Procrastination vs. celle du "Earliness Principle" (cf. Chomsky 1999), je m'abstienne tout simplement de poser la question de savoir si l'un de ces deux mouvements est plus naturel, plus économique, ou, au contraire, éventuellement plus marqué, que l'autre...
} 
Georges Rebuschi — Lapurdum 5 (2000) : Appendice

\section{APPENDICE}

Plan

0 . Présentation

1. Les RSL non-standard dans quelques traductions de St. Matthieu du $19^{\mathrm{e}}$ siècle

2. Autres exemples tirés de divers dialectes

3. Le roncalais de $P$. Hualde

4. Bilan

\section{Présentation}

Cet appendice se justifie de par le fait que le tour examiné dans l'article est totalement ignoré des descriptions grammaticales et des études linguistiques basques : il faut montrer qu'il correspond à une réalité indiscutable, en dépit de ce silence, si bien que j'en présente explicitement 116 ci-dessous. Dans la première section, je présente, et commente le cas échéant, les 57 ex. de RSL nst. relevés dans les diverses traductions de St. Matthieu qui en offrent au moins un, excepté celle de Hualde $( \pm 1860)$ en roncalais, qui sera examinée au $\S 3$, car ce texte en offre à lui tout seul la moitié du nombre donné à l'instant. Dans l'intervalle (section 2), je complète le tour des dialectes et sous-dialectes avec 31 exemples relevés au cours de lectures (un peu) plus variées. Dans la 3e section donc, j'examine les 28 occurrences relevées dans Hualde, et montre que ce qui se passe dans ce dialecte (ou idiolecte?) dépasse de manière intéressante les limites établies dans le texte principal et les deux premières sections de cet appendice.

\section{Les RSL non-standard dans quelques traductions de St. Matthieu du $19^{\mathrm{e}}$ siècle}

Grâce, en particulier, aux publications d' $O O V$ et $B O E,{ }^{42}$ il est devenu possible de travailler sur des versions dialectales distinctes des mêmes textes, ce qui compense largement le fait qu'il s'agisse de traductions (de toute manière, les textes basques non traduits sont extrêment rares avant le $20^{\mathrm{e}}$ siècle). Pour éviter d'allonger inutilement le texte, la trad. mot-à-mot n'est pas donnée, mais le marquage casuel non-standard reste signalé, ainsi que le pronom (éventuellement Ø) et l'affixe verbal absolutifs correspondants de la matrice. La référence au passage de l'Evangile est suivie du nom du traducteur, ainsi que du dialecte: voir les Références du corpus pour plus de détails ${ }^{43}$. Lorsque plusieurs traducteurs offrent une RSL nst. pour le même passage, l'ordre choisi va grosso modo des dialectes de l'est vers ceux de l'ouest.

Mt 5,19 Cette première série d'exemples est instructive, car ce verset se décompose en deux moitiés qui comportent chacune une $\mathrm{R}(\mathrm{S}) \mathrm{L}$ disloquée ${ }^{44}$. Or aucune des traductions ne comporte

\footnotetext{
${ }^{42}$ Les variantes publiées par L.-L. Bonaparte dans la $2^{\mathrm{e}}$ moitié du $19^{\mathrm{e}}$ siècle à Londres sont systématiquement signalées dans $B O E$; je n'ai donc utilisé la reproduction facsim. d'OOV (Euskaltzaindia 1991) que pour les traductions de Salaberry et d'Inchauspé, dans lesquelles j'ai pu vérifier qu'aucune RSL nst. ne figurait. La même remarque s'applique aux deux versions d'Echenique publiées respectivement par L.-L. B. et P. Salaburu (ainsi qu'au texte roncalais de Hualde, examiné au § 3).

43 Jai laissé de côté des données ambiguës, comme Mt 20,27 chez Samper (salazarais) : Eta nai duenak izan zuen artean lenbizikoa, izan bear zu zuen siervoa 'et celui qui veut être le premier parmi vous, il doit être votre esclave', car, d'une part, le déontique bear est, comme dans tous les parlés orientaux, compatible avec un aux. soit transitif soit intransitif lorsque le verbe lexical qu'il régit est inaccusatif, et d'autre part parce la forme conjuguée tronquée $z u$ peut correspondre aussi bien à l'allocutif $d u z u$ (inacc.) qu'à l'allocutif dizu (transitif). Par ailleurs, quand plusieurs passages cités pour le même verset ne divergent que sur des détails non-pertinents pour le problèmes des RSL nst., je ne donne la traduction que pour le premier d'entre eux.

${ }^{44}$ En voici la version labourdine de Harriet (1855): ${ }^{19 \mathrm{a}}$ Nork ere bada hautsiko baitu manamendu hetarik xumeenetarik ere bat, eta horrela baitaroe erakutsiko gizonei, edukia izanen da zeruetako erresuman azkentzat; ${ }^{19 b}$ bainan hek begiratuko dituena, eta begiratzen erakatsiko duena, izanen da handi zeruetako erreseman. [Voir le texte pour la traduction.] On y note le choix stylistique d'employer une RL dans la première partie, et une RSL dans la seconde, alors que le texte latin offre deux RL strictes (qui...).
} 
deux RSL nst. (comparer 5,22 plus loin); qui plus est, la répartition semble totalement aléatoire. Un autre intérêt de ce verset est que chacune des $\mathrm{R}(\mathrm{S}) \mathrm{L}$ se décompose en fait en deux sousstructures coordonnées, ce qui offre en sus des illustrations de coordination asymétriques, phénomène qui mériterait à lui tout seul une étude sérieuse, et dont on peut simplement dire ici qu'il est licite lorsque l'interprétation sémantique des deux syntagmes coordonnés est identique, indépendamment de leur forme syntaxique donc.

(A1) $5,19 \mathrm{a}-$ Echenique- $\mathrm{B} / \mathrm{E}^{45}$ (baztanais). Artakotz, nork ere austen badu manamendu ttipi otarik bat, eta ola erakusten duenak gizonei, arras ttikia deitua izain da Ø zeruetako erreinuan [...]. 'Pour cela, celui qui viole un seul de ces commandements, et enseigne cela aux hommes, on l'appellera très petit dans le royaume des cieux.'

Noter la coordination asymétrique entre une relative libre au sens strict ${ }^{46}$ et une RSL - avec, ici comme ailleurs, une interprétation non-additive.

(A2) 5,19a-Anon. A (haut-navarrais méridional). Orgatik nornaiek autxten luken mandamentu ebetaik dénik ere txikien-txikiena idurian, eta erakusten lukenak ála gizonei, anitz ttipia [...] deitua izain dá $\varnothing$ zeruen erreinuan [...].

Il y a ici une autre coordination asymétrique, maintenant entre une structure assez étrange, qui rappelle, sans leur être identique, les protases « inconditionnelles» ou concessives, et sur laquelle je ne puis m'appesantir, et une RSL : ici comme dans (A1), c'est clairement, comme il a été dit plus haut, l'interprétation sémantique identique des deux groupes coordonnés qui rend licite cette asymétrie.

Dans les ex. suivants, on notera respectivement pour (A3) et (A4) une coordination symétrique entre deux RSL nst. - je saisis l'occasion pour faire remarquer que je n'ai presque ${ }^{47}$ jamais trouvé de coordination asymétrique entre une RSL standard et une non-standard -, et une coordination avec ellipse du matériel périphérique droit dans la deuxième relative.

(A3) 5,19a-Otaegi (guipuzcoan). Eta [a]la mandamentu otakon bat ausitzen donak izan diteken txikinak iritzigaiti, ero gizonai eakusten dienak ala eiten, danetan txikintzat eukiko da $\varnothing[\ldots]$ zerutako erreinun.

(A4) 5,19a-Anon. B (guip.). Ala bada autsitzen duenak aginde txit txiki oietatikan bat, eta onela erakusten dienak gizonai, txit tkikia deitua izango da Ø Zeruetako erreinoan.

(A5) 5,19a-Udabe (guip.). Edozein askatu nai duenak manamendu txit txiki obetatikan bat, eta onela erakutsi diotet [sic] gizonai, txit txikia izango dala deitua $\emptyset$ zeruetako erreinoan.

Noter dans ce dernier ex. la présence d'un quantificateur à gauche de la RSL, construction qui revient plusieurs fois chez ce traducteur; comme il ne s'agit pas d'un élément lexical, l'analyse proposée dans le texte principal semble pouvoir être préservée, peut-être au prix de l'introduction d'une projection intermédiaire entre D' et CP (voir aussi les ex. baztanais signalés plus loin, en 2.2, qui sont pour la plupart plus complexes à traiter $)^{48}$.

\footnotetext{
45 Il existe deux versions distinctes de la trad. de Matthieu par Echenique en baztanais : la version "A/S", qui est la version A de $B O E$, publiée auparavant par Salaburu (1980), et la version "B/E", qui est celle publiée à Londres par Bonaparte en 1857, et reproduite dans Euskaltzaindia $(O O V, 1991)$; que l'une soit une correction de l'autre, ou pas, il est remarquable que chacune contienne des RSL nst., cf. (A8,9).

${ }^{46}$ Qui plus est, cette relative libre a comme $\mathrm{C}^{\circ} b a$ - 'si' plutôt que bait-ou -(e)n, comme dans cinq autres passages, phénomène qui n'est pas sans conséquence pour l'analyse des périodes corrélatives (cf. Rebuschi 2000, 2e partie, là-dessus), d'autant plus qu'il est attesté ailleurs (Ubillos 1785, guipuzcoan; Soubelet 1969, bas-navarrais) : il s'agit d'un autre tour non-standard, qui mériterait lui aussi un examen approfondi, à mettre évidemment en relation avec la coordination asymétrique signalée ici, et celle notée pour (A2).

47 Voir cependant (B18) infra.

48 Voir la note 65 . La montée postulée de l'opérateur relatif abstrait se ferait alors en deux étapes, cet élément passant d'abord par Spéc,NumP, transformant, toujours par accord spécificateur-tête, edozein, ou guztia dans (A17) etc., en un simple élément qualitatif insistant sur la non-pertinence absolue des différences pouvant caractériser les éléments de l'ensemble considéré (cf. la valeur de l'anglais any vs every). Cette question devra évidemment être reprise en détail.
} 
Georges Rebuschi — Lapurdum 5 (2000) : Appendice

Voici maintenant les deux exemples de RSL nst. concernant la deuxième partie du verset:

(A6) $5,19 \mathrm{~b}$ - Samper (salazarais). [...] Baia beiratzen tuenak eta erakusten tuenak, kori izanen $\boldsymbol{z} \boldsymbol{u}^{49}$ edokrik anditako Zeruetako erreinuan. 'Mais celui qui les observe et qui [lit. 'celui qui'] les enseigne, celui-là sera tenu pour grand dans le royaume des cieux.'

(A7) $5,19 \mathrm{~b}$ - Añibarro, p. $208^{50}$ (biscayen). [...] baña egiten dituanak, ta irakasten dituank, au andiá deituko da zeruetako erreinuan.

Mt 5,22 Contairement à ce que pourrait faire croire la répartition des RSL nst. dans le verset qui précède, on peut maintenant voir qu'il n'y a en fait nulle contrainte stylistique bloquant la répétition immédiate d'une RSL nst. derrière une autre.

$(\mathrm{A} 8,9)$ 5,22 - Echenique A/S \& B/E 51 (bazt.). Eta bere anaiai erten dionak, 'Raka', obligatua izain da $\varnothing$ kontziliora. Eta erten dionak, 'Ergela', geldituko da Ø suain gehenara obligatua. 'Et celui qui dit à son frère, "Raka", il devra passer en jugement. Et celui qui lui dit, "Sot", il sera passible du feu de la Géhenne.' 52

(A10,11) 5,22 - Anon. B (guip.). [...] Eta esaten dionak bere anaiari 'Raka', hogendun izango da $\varnothing$ kontzilioarena. Eta esaten duenak'Eroa', hogendun izango da Ø infernuko suarena.

Mt 5,32 Ce passage est le seul du type qui nous intéresse trouvé chez Harriet dans Matthieu ; s'il n'y en avait un autre (cf. B22) dans Jean, on pourrait penser à une simple coquille - mais il faut souligner que ce traducteur a présenté son texte comme une version corrigée et améliorée du ms. de Haraneder (1740), qui, n'offre, lui, aucun exemple de RSL nst. (sous réserve d'inventaire bien entendu).

(A12) 5,32 - Harriet (labourdin). [... ] eta senharrak utzia den bat emaztetzat hartzen duenak, arrotzharagitan nahasten dela $\varnothing$. '[...] et que celui qui prend pour épouse une [femme] qui a été abandonnée par son mari, il se vautre dans l'adultère.'

Mat 5,41 Ce verset présente apparemment un datif de topicalisation sur la RSL, car ni le prédicat de la relative ni celui de la matrice ne requièrent ce cas, mais il faut bien voir que le contexte, c'est-à-dire et le verset précédent, et le verset suivant, offrent des RSL disloquées dont le cas externe, repris dans la matrice, est un datif : c'est probalbement là un facteur important, s'il n'est pas tout simplement déterminant.

(A13) 5,41 Samper (salaz.). Eta prezisa zinozkenari fatra kargatrik milla urratsetan, fan zte arekin bertze bi milatan. 'Et à celui qui vous forcerait à faire mille pas chargé, faites-en deux mille avec lui.'

(A14) Mat 5,41 - Anon. A (H-N mérid.). Eta bortxatzen zaituenari joaterá kargatuik mila pausutan, zoaz areki bertze bi mila yagotan.

(A15) Mat 5, 41 - Udabe (guip.). Eta edozein kargatzen zaituenari ordu baterako, zuaz berarekin biean.

(A16) Mat 5,41 - Anon. B (guip.). Eta milla pauso kargatua egin arazten zaituenari, zoas berarekin beste bi milla geiago.

Mt 7,21 On notera ici la présence du quantifieur $g u z(t) i a$ dans la première RSL, qui occupe (peut-être) la position de $\mathrm{D}^{\circ}-$ mais voir aussi ce qui a été dit à propos de edozein dans (A5).

\footnotetext{
$49 Z u$ est la forme allocutive vouvoyée tronquée de $d u z u=d a$.

50 Jindique la page parce que les passages traduits de Matthieu (en fait, environ 95\% du texte) sont éparpillés tout au long de l'ouvrage.

${ }^{51}$ Les variations morphonologiques que présentent les deux versions sont sans pertinence pour notre propos.

52 Pour le même passage, Anon. A (HN mérid.), on a : Nornai dén artzen duena begietan bere anaiá, meregiko dú kondena dezan juezak, qui rappelle d'abord, sans la reproduire exactement, la RSL de (A5). Mais l'absence de marque ergative dans duen.a n'est pas un exemple de percolation de l'absolutif, car le prédicat de la RSL lui-même (tout comme celui de la matrice) appelle l'ergatif; il s'agit donc peut-être d'un simple lapsus calami, mais voir aussi l'ex. cité dans la note 56.
} 
Georges Rebuschi — Lapurdum 5 (2000) : Appendice

$(\mathrm{A} 17,18)$ 7,21 - Anon. A (H-N mérid.). Ez erraten didan guziak, 'Hó Jauna, Jauna!' sartuko dá orgatik zeruen erreinuan, baixik iten duenak nere Aita zerukoin borondatea, áu dá sartuko déna zeruen erreinuan. 'Ce n'est pas celui, quel qu'il soit, qui me dit "O, Seigneur, Seigneur" qui entrera pour autant dans le Royaume de Cieux, mais celui qui fera la volonté de mon père, celui-là [lit. celui-ci] est celui qui entrera dans le R. des C.'

Mt 7,24 Remarquer la RSL appositive finale en -(e)na, qui ne s'accorde pas en cas avec le DP datif auquel elle est adjointe. On retrouvera le même fait dans (A20) :

(A19) 7,24 - Udabe (guip.). Bada edozein aditzen dituenak itz obek, eta egiten, konparatuko det $\varnothing$ baroi prudente bati, egin duena bere etxea arrokaren gañean. 'Mais celui, quel qu'il soit, qui entend ces paroles, et les met en pratique, je le comparerai à un homme ${ }^{53}$ prudent, qui a construit sa maison sur le roc'.

\section{Mt 7,26}

(A20) 7,26 - Udabe (guip.). Eta edozein aditzen diskidanak itz obek, eta ez egin, konparatuko det Ø baroi ero bati egin zuena bere etxea ondarraren gañean. 'Et celui qui entend ces miennes paroles [lit. qui m'entend ces mots], et ne les met pas en pratique, je le comparerai à un homme fou qui avait construit sa maison sur le sable.'

(A21) 7,26 - Otaegi (guip.). Baño nik emate'ituan konseju ok aitu ta eiten ez ditunak ${ }^{54}$ bere exea arean gañean ein zon gizon ero baten antzekoa izango da $\varnothing$. [lit. 'Mais celui qui entend les conseils que je donne et ne les applique pas sera comparable à un homme fou qui avait construit sa maison sur le sable.'

Mt 10,22 Dans ces deux traductions, le verbe monoactanciel iraun 'durer, tenir', est inergatif et non inaccusatif (c'est ce que Lafitte 1962 appelle par antiphrase les « verbes déponents ») : il prend donc un unique argument ergatif, qui se manifeste ici de manière non-standard (voir aussi les trad. de Mt 24,13 plus bas pour un passage identique).

(A22) 10,22 - Cazenave 55 (B-N oriental). Azkenerano irauten dienak, hura salbatuko 'uzu. 'Celui qui tiendra jusqu'à la fin, celui-là sera sauvé.'

(A23) 10,22 - Anon. A (H-N méridional). Irauten duenak azken fineraño, áu salbatua izain dá.

Mt 10,32 / 37 / 38 Les exemples suivants n'ont pas besoin de commentaires particuliers.

(A24) 10,32 - Udabe (guip.). Bada edozein konfesatzen nauenak gizonen aurrean, nik ere konfesatuko det $\varnothing$ nere aita zeruetan dagoenaren aurrean. 'Donc quiconque me reconnaîtra devant les hommes, moi aussi je le reconnaîtrai devant mon père qui est aux cieux.'

(A25,26) 10,37 - Cazenave (B-N oriental). Maite dienak aita edo ama ni bano gehio, etzu Ø nitaz digno; eta maite dienak semia edo alaba ni bano gehio, etzu Ø nitaz digno. 'Celui qui aime son père ou sa mère plus que moi, il n'est pas digne de moi ; et celui qui aime son fils ou sa fille plus que moi, il n'est pas digne de moi.'

(A27) 10,38 - Anon. A (H-N méridional). Eta eztuenak artzen soñean bere gurutzea eta neri segitzen, eztá $\varnothing$ nere dignó. 'Et celui qui ne prend pas sa croix sur le dos et ne me suit pas, il n'est pas digne de moi.'

Mt 13,19 Dans ceux qui suivent par contre, on a une illustration de la non-reprise de la RSL par un argument ou circonstant de la matrice, car le pronom de rappel est au génitif, et donc interne

\footnotetext{
53 Baroi adapte évidemment ici l'esp. varón, et non barón.

${ }^{54}$ L'absence de virgule ici ne semble pas indiquer d'absence de topicalisation; voir la section 3 de cet appendice pour une discussion de cette question.

55 C'est l'orthographe employée dans BOE, Elialdeko Behe-nafarrera, vols. I \& II ; Bonaparte écrivait «Casenave».
} 
Georges Rebuschi — Lapurdum 5 (2000) : Appendice

à un DP argumental (comparer (20) dans le texte principal, et (A44) plus loin dans cet appendice) :

(A28) 13,19 - Cazenave (BN oriental). Intzuten dienak erresumako hitza, ez tienian peetxatzen izpiitu gaxtua, jiten duzu eta hartzen dizi hain bihotzian ereina dena. 'Celui qui entend la parole du royaume, et qui n'en fait rien, l'esprit malin vient et prend dans son cœur ce qui [y] a été semé.'

(A29) 13,19 Echenique B/E (bazt.). Aditzen duenak erreinuko itza eta ez duenak entenditzen, etortzen da gaistoa, eta artzen du ain biotzean erein zena. ${ }^{56}$

Mt 15,4 Voici à nouveau un cas tout-à-fait normal :

(A30) 15,4 - Udabe (guip.). Juramentatzen duenak aita edo ama, ill bedi Ø eriotzean. 'Celui qui insulte son père ou sa mère, qu'il soit mis à mort.'

MT 18,6 Dans les deux extraits suivants, on note un cas exceptionnel de substitution d'un ergatif interne à un datif externe :

(A31) 18,6 - Samper (salaz.). Baia eskandaliza lokenak txikilintto ene baitan ginesten dien kuetarik bat, obe izan liakiozu ${ }^{57} \varnothing$ eser lazoien tilintan lepotik axto batek mogitzen duen errota-arri koitarik bat, eta kala fan ladien unditzra itxasoaren barnenean. 'Celui qui scandalise [=fait tomber dans le péché] un de ces petits qui croient en moi, il serait mieux pour lui qu'on lui attache au cou une de ces meules que fait tourner un âne, et qu'ainsi il se noie [lit. qu'il aille au fond de la mer].'

(A32) 18,6 - Anon. A (H-N mérid.). Baña nere baitan sinesten duten aur tipitto ebeteik bat ere eskandalitzatzen lukenak, obeki l[a]kioke Ø lót dezoten leporá asto batek mogitzen duen errotari oieteik bat, ta alá úndi dain itxaso zolan. [id. $]^{58}$

\section{Mt 19,9}

(A33,34) 19,9 - Cazenave (B-N oriental). Bainan ere erten dauziet, uzten dienak bere emaztia, baldin ezpada adulterioko kasia, eta beste bat hartzen badu, adulterian eortzen dela ; eta utzia den emaztia esposatzen dienak, adulterian eortzen dela. 'Mais, je vous dis aussi que celui qui abandonne son épouse, s'il ne s'agit pas d'un cas d'adultère, et en prend une autre, il tombe [lui-même] dans l'adultère ; et que celui qui épouse une femme qui a été répudiée tombe [aussi] dans l'adultère.'59

Mt 20,26/27 Noter que pour ces deux passages de structure parallèle, seuls deux traducteurs, Cazenave et Udabe, emploient deux fois la forme non-standard : les autres ne l'utilisent qu'une fois, toujours de manière aléatoire, semble-t-il.

(A35) 20,26 - Cazenave (B-N oriental). [...] bainan zien artian handien izan nahiko dienak, zien zerbitzari izan dailela $\varnothing$. 'Mais celui qui veut être lez plus grand parmi vous, qu'il soit/se fasse votre serviteur.'

(A36) 20,26 - Udabe (guip.). Nai duenak zuen artean egin aundi, izango da $\varnothing$ zuen serbitzaria.

(A37) 20,26 - Añibarro, p. 345 (bisc.). Bada zuen artean nagusiago izan gura dabenák, izan bedi $\varnothing$ zuen serbitzaria.

56 Inversement, dans la trad. suivante, l'absolutif est pris comme cas par défaut: Nornai dén eitzen duená Jangoikoin edo Ebangelioin itzá, eta éz gelditzen konsideratzerá itz ónen : dátor espiritu gaixtoa, eta arrapatzen dió Ø[DAT] bere biotzean ereiki zená. (Anon. A, H-N méridional, Mt 13,19).

57 Allocutif de lekioke.

58 La trad. de ce verset par Añibarro ( \pm 1800 , bisc.) est bien plus étonnante encore, puisque qu'il n'y a pas de résurgence de l'ergatif interne, mais un marquage datif qui correspond à un argument d'une proposition enchâssée dans la matrice, et non-finie : Eta nigan sinisten daben txikitxoai bekatu-bidea emoten deutsanari, obe leuke Ø[ERG] errota arri bat bere idunera estu Ø[DAT], ta itxas-ondarrera botatea, lit. 'et à celui[DAT] qui ouvre le chemin du péché aux petits qui croient en moi, il [Ø, ERG] aurait [=ferait] mieux de [se-DAT (faire)] serrer une meule à son cou et de se [faire] jeter au fond de la mer'.

59 Anon. A (H-N mérid.) présente ici à nouveau un "oubli " d'ergatif, bien que tant le verbe de la RSL que celui de la matrice soit l'ergatif : Alá badá, eranzuten diziet ezi nórnai dén despeitzen dukena bere emaztea, non éztén andreketa kasos, eta orño kaso ontan, ezkontzen dén bertze batekin, alako onék iten dú adulterio... 
Georges Rebuschi — Lapurdum 5 (2000) : Appendice

(A38) 20,27 - Cazenave (B-N oriental). Eta zien artian lehen izan nahi dienak, zien esklabo izain duzu Ø. 'Et celui qui veut être le premier parmi vous, il sera votre esclave.'

(A39) 20,27 - Echenique A/S \& B/E (bazt.). Eta izan nai duenak lena zuen artean, izan bedi $\varnothing$ zuen zerbitzari.

(A40) 20,27 - Anon. A (H-N méridional). Eta nai lukenak izán zuen artean lendabixikoá izan dailá Ø zuen mutil.

(A41) 20,27 - Udabe (guip.). Eta nai duenak zuen artean izan lenengoa, izango da $\varnothing$ zuen serbitzaria.

Mt 21,44 Voici maintenant un cas rarement attesté, bien que logiquement aussi aisément dérivable que la percolation de la marque ergative, d'apparition d'un datif interne sur une RSL qui correspond à un argument absolutif dans la matrice :

(A42) 21,44 - Cazenave (B-N oriental). (Eta harri hartaat eortzen dena phorroskatia izain duzu, eta) gaineat juaiten zakonai, lehertia izain duzu $\varnothing$. '(Et et celui qui tombera sur cette pierre sera réduit en miettes; et) celui sur qui elle tombera, il sera écrasé' [lit. 'et à celui[DAT] qu'elle lui[DAT] ira dessus, il sera écrasé.']

Mt 23,16/18 A côté des RSL nst. ordinaires de (A43) et (A45), on trouve un cas plus intéressant de matrice sans pronom de reprise qui déclenche l'apparition de l'ergatif interne dans (A44) - cf., dans le texte principal, l'ex. (20) :

(A43) 23,16 - Añibarro, p. 372 (bisc.). [...] tenploko urrea gaiti juramentu egiten dabenák, zordun da Ø! 'Celui qui jure par l'or du temple, il est engagé.'

(A44) 23,16 - Uriarte (bisc.). Juramentu egiten daben guztijak tenplubagaitik, ez da ezer. Lit. 'Celui[ERG] qui jure par le temple, ce n'est rien.'

(A45) 23,18 - Añibarro, p. 372 (bisc.). [...] juramentu egiten dabenak aldaran dagoan ofrenda gaiti, zordun da $\varnothing$. 'Et jurer par l'autel, ce n'est rien ; mais celui qui jure par l'offrande qui est sur l'autel, il est engagé.'

Mt 23,37 Le cas suivant est intéressant, parce qu'il s'agit d'une RSL apposée à un nom propre à valeur vocative, lui-même régulièrement à l'absolutif pour cette raison même. C'est donc un autre mécanisme de non-transmission de cas par chaîne que celui traité dans le corps de l'article, qui est d'autant plus étonnant que s'il y a non-correspondance casuelle entre une RSL apposée et le DP auquel elle est apposée, on obtient usuellement une configuration dans laquelle la RSL est à l'absolutif, cf. (A19-20).

(A46) 23,37 - Echenique B/E (bazt.). Jerusalem, Profetak iltzen dituzunak, eta arrikatzen zure gana bidaliak direnak, zenbat aldiz nahi izan ditut bildu zure umeak [...] ? 'Jérusalem, toi qui tues ${ }^{60}$ les prophètes, et lapides ceux qui te sont envoyés, combien de fois ai-je voulu rassembler tes enfants?'

MT 24,13 Voici maintenant les nombreux exemples de Mt 24,13 qui reprennent 10,22, cf. (A2223). On remarquera en (A51) que le traducteur a ajouté une RSL coordonnée, qui duplique d'autant plus facilement l'ergatif que c'est le cas qui correspond aux deux positions relativisées.

(A47) 24,13 - Cazenave (B-N oriental). Bana azken pundu'ano irauten dienak, hura 'uzu ${ }^{61}$ salbatuko dena. 'Mais celui qui tiendra jusqu'au dernier moment, c'est celui-là qui sera sauvé.'

(A48) 24,13 - Samper (salaz.). Eta perseberatzen duenak finealaño, kori zu salbatren dena.

(A49) 24,13 - Echenique B/E (bazt.). Baña azkeneraño irauten duenak, ura izain da salbatua.

(A50) 24,13 - Anon. A (H-N mérid.). Irauten lukenak ordea azken fineráño, óri salbatuko dá.

\footnotetext{
${ }^{60}$ La construction de l'équivalent d'une relative qui, en français ou en anglais, serait apposée à un pronom de première ou deuxième personne est ici tout à fait normale, car les pronoms personnels ne peuvent pas prendre de relative préposée ; la forme basque est donc littéralement 'le/la qui tues / tuez les prophètes', ce qui constitue un nouvel argument en faveur d'un accord sémantique spécificateur-tête dans le DP contenant un CP, puisque le trait de personne (ici, de la $22^{\mathrm{e}}$ ), qui se manifeste sur la FVF interne au CP relatif, doit aussi être transmis de l'opérateur vide vers le dét., si le DP global doit, comme c'est usuellement le cas, s'accorder avec le prédicat de la matrice - ce qui serait le cas si la suite du passage était barkatua $\approx$ /kondenatua izanen zara 'tu seras pardonnée / condamnée'.

${ }^{61}$ Forme tronquée de $\boldsymbol{d} u z u$, allocutif de $\boldsymbol{d} a$.
} 
(A51) 24,13 - Añibarro, p. 377 (bisc.). Baña dirauanák, ta sufrietan dabenák azkeneraño, au salbauko da. Lit. 'Mais celui qui dure, et tient dans les soufrances jusqu'à la fin, celui-ci se sauvera.'

Mt 25,18 Dans (A52), le Dét. usuel - $a(\boldsymbol{k})$ est remplacé par un démonstratif; comme le dialecte est (sud) oriental, rien ne s'oppose à le considérer comme occupant effectivement la place du $\mathrm{D}^{\circ}$ (cf. (13a) dans le texte principal) - mais bien entendu, comme le montrent (A53-54), cela ne change rien à nos données :

(A52) 25,18 - Samper (salaz.). Baia errezibitu zuen karek bat, fan zinuen ${ }^{62} \varnothing$ [...]. 'Mais celui(-là) qui [en] avait reçu un [=un talent], il s'en alla.'

(A53) 25,18 - Anon. A (H-N mérid.). Errezibitu zuenak órdea bát, joan zé Ø [...].

(A54) 25,18 - Udabe (guip.). Baña bat errezibitu zuenak, joan zan Ø [...].

Mt 26,23 Les deux exemples suivants sont, plus encore que les précédents, d'interprétation nettement spécifique, ce qui montre bien que la spécificité d'une RSL est bien à distinguer des cas d'ellipse proprement dite.

(A55) 26,23 - Samper (salaz.). Sartzen duenak enekin bere eskua platerean bustitzeko ogia, kori zu traidorea. 'Celui qui met la main dans le plat avec moi pour tremper son pain, c'est lui le traitre.'

(A56) 26,23 - Anon. A (H-N mérid.). Sartzen duenák nereki bere eskua platereán, ogiain bustitzeko, óri dá traiziazaleá.

Mt 26,48 Pour finir, voici enfin un dernier exemple de percolation vers le haut du DP d'une marque interne de datif, celle portée par l'opérateur relatif abstrait qui s'accorde avec la FVF, cf. le -o-de l'aux. dioda(nari):

(A57) 26,48 - Anon. A (H-N mérid.). Nik ápa ematen diodanari, óri dá ; segura zazié. 'Celui[DAT] à qui je donnerai un baiser, c'est lui ; saississez-le.'

\section{Autres exemples tirés de dialectes divers}

Certains dialectes, ou plutôt certains idiolectes spécifiques à certains dialectes, sont très bien représentés dans la section qui précède; mais en fait, seul le guipuzcoan est représenté par trois variétés, et trois traducteurs différents ; par contre, nous n'avons rien en souletin (le ms. d'Inchauspé n'est pas disponible à l'heure où j'écris ces lignes, et l'on sait que les versions publiées à Londres par L.-L. Bonaparte ont souvent été remaniées), rien en mixain (parler intermédiaire entre le souletin et le bas-navarrais oriental), rien en aezcoan, etc. Il est donc utile de compléter les données qui précèdent de quelques autres, afin de montrer qu'il n'existe aucun dialecte qui ignorerait totalement les RSL non-standard, et que ces RSL ne sont pas forcément le produit d'idiolectes particuliers. (Pour changer un peu la présentation, on vas maintenant aller d'ouest en est.)

\subsection{Le domaine centro-occidental}

Il existe depuis longtemps une tradition, chez les auteurs biscayens, d'écrire en guipuzcoan. C'est le cas de J. A. Mog(u)el, qui a rédigé un long catéchisme, Cristau eracasle euscalduna, vers 1802 (cité d'après l'éd. de 1994); plusieurs passages contiennent des RSL avec un ergatif interne externalisé, ce que note bien l'éditeur dans l'introduction, mais sans en donner la liste exhaustive. On y trouve ainsi des ex. comme les suivants, dont (B3), traduit de St. Luc, et les autres, qui ne semblent provenir d'aucune traduction :

(B1) $\operatorname{Mog}(\mathrm{u}) \mathrm{el} 1802$, p. 145. [...] juramentu egiten duenak [...], jartzen da Ø oitura arturik [...]. 'Celui qui fait un serment en prend l'habitude', lit. '... il s'installe[INAC] ayant pris l'habitude.'

62 Forme allocutive de zen, AUX. inacc. au passé, 3e p. sg. 
(B2) $\operatorname{Mog}(\mathrm{u}) \mathrm{el} 1802$, p. 238. Utsegiten duenak [...] peligro andian arkitzen da $\emptyset$. 'Celui qui se trompe, il se trouve[INAC] en grand danger.'

(B3) $\operatorname{Mog}(\mathrm{u}) \mathrm{el} 1802$, p. 289 (cf. Lc 14,26). Gorroto ez dionak guraso, edo aideari, ez da $\emptyset$ nere eskolakoa. 'Celui qui n'a pas de haine envers ses parents et ses frères, il n'est pas mon disciple.'

(B4) $\operatorname{Mog}(\mathrm{u}) \mathrm{el} 1802$, p. 333. Zenbat prestuago da daukana etxean bear dan gari ta ardoa, ... ta dakianak non gastatu ta non ez. 'Combien plus sage est celui qui garde chez lui le blé et le vin qu'il faut, et qui sait quand en user et quand s'abstenir !'63

Ce dernier passage est un des très rares exemples de RSL nst. que j'aie trouvés avec un verbe fort, conjugué synthétiquement, autre qu'avoir' ; on remarque aussi que la RSL nst. est à droite du prédicat inaccusatif; ici, on peut penser que la percolation de l'ergatif interne vers la périphérie droite du DP est liée à l'ellipse du prédicat, non répété, mais on verra plus loin que d'autres explications, moins ad hoc, sont possibles.

Un autre texte intermédiaire entre le biscayen usuel et le guipuzcoan est fourni par le long ms. d'A. de Aguirre (1812-1814), rédigé dans la variété orientale de biscayen telle qu'elle est parlée à Oñate (Guipuzcoa) et publié dans BOE, Bizkaiera, vol. 2. Ce texte présente un nombre extraordinaire d'omissions du suffixe ergatif sur des $D P$ à tête lexicale lorsque le contexte requiert ce cas : j'en ai compté 27 , et cela rend d'autant plus remarquable le fait que des RSL nst. avec marque externe d'ergatif interne s'y trouvent, alors que l'inverse n'y est pas attesté. En voici un ex. :

(B5) Aguirre 1812-14, p. 623. Proximoa biotzetik amatsen esduenak, illik dago Ø animan. 'Celui qui n'aime pas [son] prochain [du fond] de son cœur, il est mort dans [son] âme.'

\subsection{Le baztanais}

Le baztanais est certainement le (sous-)dialecte qui offre le plus grand nombre d'anomalies dans les constructions relatives (semi-)libres et apparentées par rapport aux variétés de basque qui ont une tradition littéraire : on en a rencontré divers exemples dans les extraits d'Echenique dans la section précédente. Cela dit, on dispose aussi d'un texte rédigé par un auteur (voire plusieurs) assurément différent(s), quoique non-identifié(s), le long catéchisme anonyme publié dans BOE, Iparraldeko Goi-nafarrera, vol. 2 (687-988). En voici quelques exemples ${ }^{64}$.

(B6) p. 816. [...] sobera unione duenak jende soberbioekin, eginen dela bera soberbio. '...que celui qui a trop de rapports avec les gens orgueilleux deviendra lui-même orgueilleux'

(B7) p. 841. Jaten duenak ogi untarik, biziko da Ø sekulan. 'Celui qui mange de ce pain, il vivra éternellement.'

(B8) p. 852. Bere burua ezagutzen ez duenak, bestia ó animalea baño gutiago da $\varnothing$. 'Celui qui ne se connaît pas lui-même, il est moins qu'une bête.'

(B9) p. 910 [...] eskalera untan ez dakienak ongi oña firme paratzen, eroriko da Ø infernuko zuloan. 'Celui qui ne sait pas bien se tenir de peid ferme sur cette échelle, il tombera dans l'abîme de l'enfer.'

Voir encore les p. 857-8, 869, 880, 901, 924, 932, 935... Plus intéressant est l'extrait suivant :

(B10) p. 815 Gure Jangoikoak dio, edozein azerretzen [sic] dena o kejatzen dena bere anaiaren kontra, meretxiko duela juizioko tribunaleaz kondenatua izatea. 'Notre Dieu [nous] dit que celui, quel qu'il soit, qui se fâche ou se met en colère contre son frère, méritera d'être condamné par le tribunal.'

Cet ex. est, avec celui de Lizarrraga noté en (B14), l'une des très rares occurrences de RSL nst. marquées extérieurement par un absolutif déterminé par le prédicat interne à la relative, la matrice exigeant par contre un ergatif (voir la discussion dans le texte principal, $\S 4.5$ ). Noter

\footnotetext{
63 Noter encore qu'un correcteur ultérieur anonyme a ajouté un - $k$ ergatif non-requis, donc non-standard, à un DP absolutif du ms. correspondant à une RSL standard contenant un verbe transitif, Ona emen gertaera asko erakasten duenak gauza onetan 'Voici quelque chose qui [lit. 'ce qui'] explique beaucoup d'événements dans ce domaine' (p. 245 et tableau des corrections, p. 351, réf. à la p. 261, 1.14 du ms.).

${ }^{64}$ Ce texte présente bien d'autres curiosités, même dans le domaine des RL et des RSL, mais il me faut laisser pour un autre travail l'examen de ces données ; voir cependant l'ex. cité dans la note suivante.
} 
aussi que le texte baztanais cité ici offre de nombreux tours avec edozein en tête d'une RSL, mais, contrairement à ce qui se passe chez Udabe, ce quantificateur porte très souvent aussi luimême la marque casuelle interne, ce qui interdit de les traiter comme des RSL nst. ordinaires ${ }^{65}$. Pour en terminer avec ce texte, l'extrait suivant est un cas d'ergatif interne remplaçant un datif externe :

(B11) p. 864. Maite duenak itzusia, iduri zaio ederra Ø. '[A] celui[ERG] qui aime le laid, il lui semblera beau'

\subsection{Le haut-navarrais méridional}

Les illustrations des RSL nst. en H-N méridional données au $§ 1$ sont dues à un traducteur inconnu. Or ce même dialecte est surtout connu par les très nombreux écrits de Joaquín Lizarraga, dit d'Elcano, qui passe pour un écrivain très soigneux - et il est vrai que la lecture (rapide, je le reconnais) que j'ai effectuée d'un grand nombre de ses sermons n'a fourni aucun ex. de RSL nst. Cependant, la traduction du seul Evangile qu'on lui connaisse, celui de Jean, nous en offre deux parfaitement ordinaires, et un cas inverse et tout à fait exceptionnel, où c'est un absolutif interne qui remplace un ergatif attendu de l'extérieur. Les voici :

(B12) In 3,21. Baña egiten duénak égia edo zuzenbidea ${ }^{66}$, eldu dá $\varnothing$ argirá [...] 'Mais celui qui pratique la vérité ou le droit, il vient à la lumière.'

(B13) Jn 7,18. Baña billatzen duénak biali nauenaren gloria, ura dá egiáskoa [...] 'Mais celui qui recherche la gloire celui qui m'a envoyé, celui-là [it. 'celui-ci'] est vrai.'

(B14) Jn 15,5 Ni naiz aiéna, zuek zarmenduak. Mantenitzen déna ni baitan, eta ni bera baitan, onék dakárra fruitu anitz [...] 'Moi je suis le cep, et vous, les sarments. Celui qui demeure en moi, en moi en lui, celui-là [lit 'celui-ci'] porte beaucoup de fruits.'

On a manifestement ici un effet stylistique, dû au contexte : si la forme attendue, denak, avait été employée, on aurait perdu la symétrie entre les formes respectives de 'lui en moi, et moi en lui', car *(eta) nik bera baitan n'aurait aucun sens; en d'autres termes, cette nécessité de parallélisme morphologique a conduit le traducteur à actualiser une virtualité de langue dont il n'use en fait que très peu.

Notons encore l'ex. suivant, beaucoup plus ancien, trouvé dans un document de l'ouest de la Navarre, et dans lequel l'ergatif interne a supplanté le datif externe (on attendrait en effet du.en.a.ri 'à celui qui...' ; le datif de l'argument de la prop. principale est par ailleurs donné par le suffixe -o- de la forme verbal fléchie (ba)lem.o.ke) :

(B15) Haut-Navarrais d'Urdánoz, 1687 (anon., cité par Satrústegui 1981, p. 101) Eliza sandu ontan esku egosten duenak Jainkoak balemoke Ø kastigu. Lit. 'Celui[le-ERG] qui porte [la] main [sur quelqu'un] en cette sainte église, Dieu peut / pourra bien lui donner [un] châtiment.'

\subsection{L'aezcoan}

C'est, on l'a dit, le seul dialecte dans lequel L.-L. Bonaparte n'ait pas fait faire de traduction d'un Evangile. Mais on trouve des RSL nst. dans diverses traductions du catéchisme d'Astete publiées dans BOE, Aezkera (1997). Je n'indique ici, en anticipant un peu sur la section 3, que les cas les moins usuels.

\footnotetext{
${ }^{65}$ En voici un ex. parmi de nombreux autres, p. 798 : Edozeñek peleatzen duenak Yangoikoaz, ez dadiela sartu $\varnothing$ munduko egitekoetan. 'Celui, quel qu'il soit, qui se bat pour Dieu, qu'il ne se mêle pas des affaires du monde'. Le problème théorique, du point de vue « minimaliste » est évidemment que, si l'opérateur passe par Spéc, $\mathrm{NumP}$ et donne à $\mathrm{Num}^{\circ}$ son cas ergatif, on voir mal comment il pourrait continuer son chemin et le transmettre à nouveau au $\mathrm{D}^{\circ}$, puisque que trait casuel n'est pas interprétable en Forme Logique.

66 Ces deux derniers mots ne figurent pas dans le texte de Londres, 1868, mais seulement dans le ms. publié dans le vol. pertinent de $B O E$.
} 
(B16) Orbarako Dotrina-2 (p. 41). Zer eskatzen duzu petizio gortan? - Jangoikoak barka dazkigula guri gauren zorrak ala nola guk barkatzen baiteztegu gu injuriatu eta agrabiatu gituztenek. 'Que demandez-vous par cette prière? - Que Dieu nous pardonne nos fautes de même que nous pardonnons à ceux[ERG] qui nous ont injuriés et offensés'

On remarque deux choses ici : d'une part, la RSL nst. semble bien être en position argumentale, à droite du prédicat verbal, et, surtout, l'ergatif pluriel s'est substitué à un datif demandé par ce prédicat.

Dans l'ex. suivant, on note que la réponse fournie par la RSL nst. ne reprend pas une demande d'identification d'un individu : on retrouve donc approximativement le même cas de figure qu'en (A44) supra.

(B17) Garraldako Dotrina-1 (p. 68). Zer da juramentatzea egieik gabe? - Juramentu eiten duenak berak sentitzen duenain kontra, edo gezurrez. 'Qu'est-ce que jurer sans vérité ? - [Lit. ] Celui[ERG] qui fait un serment contre ce qu'il ressent lui-même, ou par mensonge.'

A la page suivante,on trouve un nouveau cas recencé de remplacement d'un ergatif externe par un absolutif interne :

(B18) Garraldako Dotrina-1 (p. 69). Nork santifikatzen 'tu xeiak? - Meza enteroki entzuten duenak, eta trabajatzen eztena xei egunetan nezesidadeik gabe. 'Qui respecte les fêtes? — Celui qui entend la messe en entier, et (celui) qui ne travaille pas sans nécessité les jours de fête.'

(Ici, on aurait attendu trabajatzen ez denak, puisque l'interrogatif est à l'ergatif). On trouve trois ex. du même type 9 pages plus loin, à ceci près que la RSL nst. à l'absolutif est maintenant normalement disloquée à gauche (mais je n'en donne qu'un) :

(B19) Garraldako Dotrina-1 (p. 78). Eta erori dena bekatu mortalean, nola disponitu bear du komekatzeko? 'Et celui[ABS] qui est tombé[INAC] dans le péché mortel, comment doit-il faire pour communier?'

Voici pour finir un nouveau cas d'absolutif fourni dans une réponse à une question ouverte dont l'interrogatif est à l'ergatif - remarquer que si la locution juramentu egin est normalement transitive, le verbe juramentatu est, lui, systématiquement inaccusatif dans ce dialecte (au moins) ${ }^{67}$.

(B20) Garraldako Dotrina-2 (p. 114). Nork faltatzen dako bigarren mandamentu gorri? — Juramentatzen dena egiarik gabe. 'Qui[ERG] manque à ce second commandement? Celui[ABS] qui jure[INAC] sans vérité.'

Des exemples plus ordinaires de RSL non-standard peuvent être trouvés au moins aux pages suivantes du volume examiné: pp. 64 (2 ex.), 70, 76, 78, 79, 93, 94.

\subsection{Le salazarais}

Outre le texte cité supré de Samper, le vol. de $B O E$ consacré à ce dialecte contient divers catéchismes. Voici un ex. de RSL nst. tiré d'un texte (rédigé par un auteur distinct), où la RSL devrait reprendre l'absolutif de l'interrogatif précédent nor (par ailleurs, il est difficile de savoir si la coordination est additive ou non ici).

(B21) Juanco, 1834 (p. 257 de BOE, Zaraitzera). Nor juaten da konfesatzera dolore eta proposito egiazkoa gabe? - Eztuenak aborrezitzen bekatua eta igesi egiten bekatuaren peligrotik. 'Qui[ABS] $\mathrm{va}[\mathrm{INAC}]$ se confesser sans véritables remords et [sans] bonnes intentions? - Celui[ERG] qui ne hait pas le péché et qui ne fuit pas le danger du péché.'

\subsection{Le labourdin}

\footnotetext{
${ }^{67} \mathrm{Ce}$ qui fait la force de tels exemples, c'est que si le verbe de la relative est transitif, et que l'opérateur y correspond au sujet, il n'y a pas d'oubli du suffixe ergatif final sur la RSL !
} 
De tous les dialectes basques, c'est celui qui a la plus longue tradition littéraire, et c'est aussi celui qui présente le moins de RSL nst. En fait, à part l'ex. de Harriet pour Mt 5,32, cité en (A12), je n'ai relevé que le suivant ${ }^{68}$.

(B22) Jn 14,269 (Harriet, 1855). Ene agintzak badakizkienak, baldin begiratzen baditu, hura da ni maite nauena. 'Celui qui connait mes ordres, s'ils les applique, c'est lui qui m'aime.'70

\subsection{Le bas-navarais}

Outre les extraits fournis de la trad. de Matthieu par Cazenave, mentionnés dans la section 1 de cet appendice, j'ai relevé trois exemples, intéressants à divers titres, dus à un auteur assez réputé, E. Zubiri "Manezaundi", originaire de Valcarlos - Luzaide. Tout d'abord, ils sont tirés d'articles de journaux qui ont été écrits entre 1928 et 1936, puis republiés par M.R. Pagola en 1990. Ils représentent donc un état de langue beaucoup plus moderne que les autres cités dans cet appendice. Par ailleurs, le premier cité ici a été corrigé, sans que cela soit mentionné, dans la version publiée par Irigaray (1958, p. 17, qui propose zuena). Troisièmement, le second et le troisième (tirés de textes absents dans le recueil d'Irigaray ${ }^{71}$ ) présentent la particularité d'offrir une percolation d'un trait d'ergatif pluriel, ce qui est, pour diverses raisons, naturellement plus rare $^{72}$. Quatrièmement, le second, (B24), présente simultanément un trait de personne dans le spécifieur de DP, puisque tant le verbe de la RSL que celui de la matrice s'accordent en (première) personne. Enfin, le troisième ex. semble présenter la RSL en position argumentale, mais il est possible de l'interpréter comme disloquée à droite, $\mathrm{cf}$. la traduction proposée.

(B23) Ehiaralarren sortua zen (Ø), Luzaidetik hurbil, eskuaraz lehenbiziko liburua idatzi zuenak, «Linguce Vascomum Primitice » deithua. 'Il était né à Saint-Michel, non loin de Valcarlos, celui [lit. le-ERG] qui écrivit le premier livre en basque, intitulé $L V P . '$ (p. 104).

(B24) Adin puska bat dugunek, Iruñeko lehengo bizitzetik oroitzen girelarik, baldintzen gira $\varnothing$ ikhustean nola [...]. 'Nous [lit. les-ERG] qui avons un certain âge, quand nous nous rappelons la vie ancienne Pampelune, nous nous étonnons[MEDIO-PAss] de voir comment...' (p. 223)

(B25) [...] Nahi izan zien bere begiez frogatu erraiten zakotena, ustez ez othe ziren gezurtari batzu haundikeria horiek salatzen zituztenek. 'Il voulut vérifier de ses propres yeux ce qu'on lui disait, se demandant si ce n'était pas [lit. s'ils n'étaient pas] des menteurs, ceux[lit. les-ERG] qui lui révélaient ces prétendus hauts-faits.' (p. 49).

\subsection{Le mixain}

Le dialecte d'Oyhénarte (1657-1664) est à la limite entre le bas-navarrais oriental et le souletin. Son recueil ne contient qu'un seul exemple de RSL non-standard, le $\mathrm{n}^{\circ} 517$.

(B26) Etxea urra zezanak egur egiteko, xaz bero zedin $\varnothing$ aurten hotzez hiltzeko. 'Celui [lit. le-ERG] qui détruisit sa maison pour faire du bois, il se chauffa[INAC] l'an passé pour mourir de froid cette année' 132) :

Dans le même sous-dialecte mixain, j'ai encore relevé l'ex. suivant, chez Lopez (1782, p.

68 On pourrait peut-être aussi signaler Mt 12,30, indiqué dans la liste d'errata à la fin du livre comme devant ne pas prendre de suffixe ergatif ; le corps du texte donne en effet Enekin biltzen eztuenak, barraiatzen ari $d a$ 'Celui qui ne rassemble pas avec moi, il s'affaire [INAC] à disperser.'

${ }^{69}$ On pourrait peut-être aussi signaler Mt 12,30, indiqué dans la liste d'errata à la fin du livre comme devant ne pas prendre de suffixe ergatif; le corps du texte donne en effet Enekin biltzen eztuenak, barraiatzen ari da 'Celui qui ne rassemble pas avec moi, il s'affaire [INAC] à disperser.'

${ }^{70}$ Le positionnement de la RSL à gauche du Spéc, CP baldin indique que celle-ci n'est pas en rapport avec le prédicat transitif de la protase condionnante.

${ }^{71}$ En fait, le recueil d'A. Irigaray (1958) contient bien aussi un article intitulé 'Iruñeko itzulea', mais il est extrêment différent, et ne contient pas l'équivalent de la phrase citée en (B25) .

${ }^{72}$ Les RSL au pl. sont, et de loin, beaucoup moins usitées que les RSL au sg. ; qui plus est, dans les dial. centraux et occidentaux, le pluriel est en -ak tant à l'ergatif qu'à l'absolutif, ce qui neutralise évidemment l'opposition recherchée. 
(B27) Abildu nahi dienak zerbait jakitaten, edo zerbait ofiziotan, etzu $\varnothing$ hasten gauza baten egun batian ika[s]ten, eta biharamunian beste baten. 'Celui[ERG] qui veut[+TR] s'exercer en quelque art [lit. connaissance] ou quelque métier, il ne commence[INTR] pas par apprendre une chose un jour, et une autre le lendemain. 73

\subsection{Le souletin}

Le seul auteur souletin chez qui j'aie trouvé des RSL nst. est Eguiatéguy (1785). En voici quelques-unes ${ }^{74}$. Dans la première, on retrouve la percolation de l'ergatif interne dans une RSL clairement disloquée à droite (par mouvement d'extraposition ou non, peu importe), mais les deux suivantes sont tout-à-fait ordinaires :

(B28) (p. 129) Ene semia, gizon baten seme hiz, dakianak irainguen edo hiltzen. 'Mon fils, tu es un fils de noble, qui sais résister ou bien mourir.'

(B29) (p. 135) Mehatxatzen dianak makhikaz, ezpataz ta süiaz[,] ohart bedi Ø ehün beso eztütiala ta bizi bat baizik. 'Celui qui menace par bâton, l'épée ou le feu, qu'il se souvienne qu'il n'a pas cent bras, et [qu'il n'a] qu'une vie.'

(B30) (p. 199) Errelijionerik eztianak, da Ø düda gaberik Jinkoaren ta jenden etsaia [...] 'Celui qui n'a pas de religion, il est sans aucun doute l'ennemi et de dieu et des hommes...'

\subsection{Le roncalais}

C'est le seul dialecte qui n'ait pas été mentionné jusqu'ici, non seulement parce que la principale source de nos connaissances dans ce parler, aujourd'hui éteint, est constituée par les textes d'un seul et même auteur, P. Hualde - dont nous allons nous occuper de manière détaillée dans la 3e partie de cet appendice -, mais aussi parce que la fréquence des RSL nst. est extraordinairement élevée chez lui. Cependant, pour faire la transition, j'offre pour conclure ce parcours inter-dialectal un autre ex. de RSL nst. en roncalais, le proverbe $n^{\circ} 263$ du recueil d'Azkue (1942) ${ }^{75}$ :

(B31) Txutx egiten dionak zeuriari, beitartera erorten zau berari. 'Celui[ERG] qui crache (lit. 'fait crachat'] au ciel, ça lui retombe sur [son] visage à lui.'

Remarque : ici encore, c'est un datif qu'on attendrait, cf. l'aux. zau, basque unifié zaio, et l'emphatique au datif berari.

\section{Le roncalais de $P$. Hualde}

On l'a dit, Les 28 exemples de RSL nst. fournis par la traduction de St. Matthieu par P. Hualde (soit la moitié du total des exemples relevés dans les autres traductions citées dans la première partie !) présentent des caractéristiques qui mettent ce dialecte, aujourd'hui éteint, un peu à part. En effet, un assez grand nombre des ex. relevés s'écartent du modèle non-standard canonique décrit dans le texte principal, et généralement illustré dans les précédentes sections de cet appendice. On peut classer les tours relevés comme suit : (i) ceux qui correspondent à la structure canonique (marquage ergatif sur une RSL disloquée reprise par un pronom visible ou

\footnotetext{
73 Remarques. (i) Le verbe hasi 'commencer' est inaccusatif dans tous les dialectes basques ; (ii) la forme etzu est la forme tronquée de ez duzu/eztuzu, allocutif poli (employé tout au long du livre) de da, aux. de 3 e p. sg.

${ }^{74}$ L'édition de Peillen (1983) comporte exactement 2000 (deux mille) notes, mais, sauf omission involontaire de ma part, aucune ne signale le caractère « anormal » des RSL non-standard, ce qui semble bien indiquer qu'aux yeux et oreilles d'un souletin, ces tournures sont tout-à-fait normales, tout comme dans le dialecte roncalais, parlé immédiatement de l'autre côté de la frontière...

75 Aucun exemple de RSL non-standard n'apparaît dans les proverbes cités comme relevant d'autres dialectes - mais on sait qu'Azkue avait la manie de «normaliser » ses données de terrain ; ne faut-il donc pas voir dans cet exemple la reconnaissance d'un phénomène somme toute normale en roncalais à ses yeux?
} 
non à l'absolutif), (ii) constructions avec positionnnement de la RSL à droite de la RSL, (iii) autres cas.

3.1. Le premier groupe, comme annoncé, comprend les RSL nst. ordinaires, et n'offre pas d'intérêt particulier.

(C1) 5,19 [...] baia guardatan eta erakustan tionak, kori izanen da ekunik anditako zeurien reinuan. 'mais celui[+ERG] qui les observe[+TR] et les enseigne[+TR], celui-là[ABS] sera[INAC] tenu pour grand dansle royaume des cieux.'

(C2) 5,21 Entzunik zaudei erran zela [...] il 'ezanak ${ }^{76}$ izanen dela Ø kondenatruk [...] 'Vous avez entendu dire que celui[ERG] qui tue[+TR] sera[INAC] condamné,77

(C3) 5,22 [...] Baia dei lazanak fatuo, izanen da Ø[ABS] iburniko suaren reo... 'Mais celui[ERG] qui l'appellera[+TR] "sot", il sera passible du feu de l'enfer.'

(C4) 7,21 [...] beizik egiten dionak ene Aita zeurian dagonaren borontatia, kori da sarten dena zeurien reinuan. 'mais celui[ERG] qui fait la volonté de mon père qui est au ciel, celui-là[ABS] est[INAC] celui qui entrera dans le royaume des cieux.'

(C5) 10,39 [...] eta gal 'ezanak bere bizia ene amorioz, bueltaten da Ø erdeitra. 'Et celui[ERG] qui perd sa vie par amour de moi, il la retrouve', lit. '... il[ [ABS] retourne[INAC] à la trouver. ${ }^{78}$

(C6) 15,4 Maradika lezanak aita edo amari, izan deila Ø kondenatruk iltra. 'Celui[ERG] qui maudit son père ou sa mère, qu'il soit[INAC] condamné à mourir'

(C7) 23,16 Baia juramentu egiten dionak tenpluaren urriaz, obligatruk dago Ø. 'Mais celui[ERG] qui jure par l'or du temple, il[ABS] reste[INAC] engagé.'

(C8) 23,18 Baia juramentu egin lezanak oberta aren gainian ixariaz, egiten da $\varnothing$ zordun. 'Mais celui[ERG] qui jure prar l'offrande qui est dessus [=sur l'autel], il se retrouve tenu.' - lit. 'il[ABS]se rend[INAC/MEDIO-PASS] endetté]'

(C9) 25,16 Errezibitu zionak borz deuri fan zen [... $]^{79}$ 'Celui[ERG] qui avait reçu cinq talents s'en alla'

(C10) 25,18 Baia errezibitu zionak bat, fan zen $\varnothing$ [...] 'Mais celui[ERG] qui [en] avait reçu un, il[ABS] partit[INAC]'

(C11) 26,23 Sartan dionak enekin bere eskiua platerian ogia bustitako, da traidora Ø. 'Celui[ERG] qui met la main dans le plat avec moi pour mouiller (son) pain, c'est[INAC] le traître.'

3.2. Le texte examiné ici présente un assez nombre identique de RSL nst. qui figurent $\grave{a}$ droite du prédicat, ce qui n'est pas sans soulever quelques questions par rapport au système dévreloppé dans le corps de l'article. Je vais donc essayer de montrer que la plupart d'entre eux (mais pas tous...), sont malgré tout compatibles avec une analyse dans laquelle la RSL est en position non-argumentale.

Dans toute la série suivante, on peut considérer que d'une part l'absence de copule (qui entraine, comme c'est généralement le cas dans les dialectes orientaux, l'absence simultanée de marque de nombre sur le prédicat), et d'autre part le positionnement topicalisé dudit prédicat, permettent de considérer que les RSL ne sont pas véritablement en position argumentale, mais dans une position de topique léger inférieure à celle du focus — cf. Rizzi (1997), qui propose

\footnotetext{
${ }^{76}$ Hualde, comme d'autres auteurs navarrais, emploie fréquemment une forme subjonctive dans les RSL, mais cela ne change rien au phénomène, cf. le passage précédent, où on est à l'indicatif. De même, en biscayen, on note qu'un auteur comme Kerexeta (1976) emploie souvent le subjonctif dans les RSL, mais n'utilise jamais le marquage casuel non-standard, et, inversement, qu'Añibarro $( \pm 1800)$, qui n'emploie pas le subjonctif dans les RSL, offre quelques exemples de d'ergatif interne extériorisé, cf. la section 1 supra.

77 Noter l'absence de virgule, qui correspond apparemment à une topicalisation légère (non contrastive); voir aussi l'alternance entre (C9) et (C10), et la discussion qui vient en 3.2.

${ }^{78} \mathrm{Si}$ le tour avec bueltatu est un calque évident de l'espagnol, il permet à la RSL nst. (qui ne saurait, elle, avoir un quelconque rapport au castillan), de se manifester.

79 Voir la note 77 : la RSL représente ici un topique léger, non-contrastif, qui s'oppose à celui de l'ex. (C10), qui est nouveau dans la narration, est donc minimalement contrastif, et se détache par suite prosodiquement, comme l'indique la virgule.
} 
explicitement qu'en italien, le FocP soit entouré de TopP récursifs. C'est aussi ce qui permet d'expliquer que les ex. (C2) et (C9) supra aient été traités comme des cas ordinaires.

(C12) 5,5. Bienabenturatu nexar egiten deinek. 'Bienheureux ceux[ERG] qui pleurent [lit. 'font larme']'

On attendrait un suffixe d'abs. pl., donc deinak. Il en va de même pour les versets 6, 8, 9 et 10 du même chapitre, construits selon un schéma identique ${ }^{80}$.

(C13) 5,6 Bienabenturatu gose ta egarri deinek justiziaz [... ' 'Bienheureux ceux[ERG] qui ont[TR] faim et soif de justice.'

(C14) 5,8 Bienabenturatu biotza argi deinek [...] 'Bienheureux ceux[ERG] qui ont[TR] le cour pur.'

(C15) 5,9 Bienabenturatu bakia prokuratan deinek [...] 'Bienheureux ceux[ERG] qui recherchent[TR] la paix'

(C16) 5,10 Bienabenturatu persekuzione sufritan deinek justiziarengatik [...] 'Bienheureux ceux[ERG] qui endurent[TR] la persécution pour la justice.'

Relèvent du même paradigme les extraits suivants :

(C17) 11,6 Eta bienabenturatu arten ez dionak eneganik eskandalozko okasionerik. 'Et bienheureux celui[ERG] qui ne trouvera[TR] pas en moi d'occasion de chute [lit. 'scandale'].'

(C18) 7,14 [... $]^{81}$ Eta zer txiki da dra arekin kausitan deinek. 'Et combien peu nombreux sont ceux[ERGPL] qui le ${ }^{82}$ trouvent.' [le = le bon chemin]

La structure exclamative de ce passage nous renvoie probablement à un autre cas de topicalisation légère de la RSL, qui se trouverait donc dans une dans une position plus basse que celle du focus, mais cependant non-argumentale, comme en (C12-17).

Les trois extraits suivants ne sont cependant pas passibles du même « sauvetage » que les précédents :

(C19) 25,20 Eltan zelarik errezibitu zionak borz deuri [...]. 'Quand vint celui qui avait reçu cinq talents...'

(C20) 25,22 Eltu zen gero bi deuri errezibitu zionak eta erran zion [...] 'Arriva ensuite celui qui avait reçu deux talents, qui dit ...'

(C21) 25,24 Azkenik, eltian errezibitu zionak deuri bakotx bat. 'Enfin, quand arriva celui qui avait reçu un seul talent.'

Ces trois ex. font partie de la même narration que (C9) et (C10). Comme il n'y a plus de thématisation du tout, il faut reconnaître que nous avons affaire ici à des exceptions claires à la condition de dislocation des RSL, déjà assouplie dans cet appendice à une plus simple condition de thématisation " légère" - mais n'est-ce pas précisément parce qu'un auteur comme Hualde utilise beaucoup les RSL nst. qu'il en vient à en systématiser le fonctionnement, et donc à relâcher les contraintes qui pèsent plus usuellement sur leur distribution ? (voir cependant les ex. (A48) à (50) de la première partie).

3.3. Il nous reste sept exemples qui ne rentrent pas, du moins directement, dans le cadre construit ici. En (C22), la RSL est en apposition à un DP absolutif, et celle de (C23) est une appositive extraposée. Ce qui fait difficulté, c'est que la tendance est que les RSL appositives prennent le même cas que le DP auquel elles sont adjointes. De plus, quand ce n'est pas le cas, c'est alors l'absolutif qui est normalement employé - on a donc violation de ces deux règles générales, puisque tant la reproduction du cas du DP ainsi modifié que la règle d'application du cas par défaut, devraient mener à l'apparition de formes absolutives.

\footnotetext{
${ }^{80}$ Les éd. de BOE, Erronkariera (1997) signalent en note que la version partielle de ce ms. publiée par Urquijo dans $R I E V$ en 1934 et 1935 avait été « corrigée » en deinak, du moins pour les versets 5,6 et 5,9. Remarquer aussi que le ms. de la trad. du catéchisme d'Astete par Hualde comporte, pour les mêmes passages, respectivement, deinak en 5,5 à 5,8, mais deinek pour 5,9 et 5,10 uniquement (selon BOE, ibid.) — alors que le texte imprimé à Londres en 1869 est systématiquement corrigé (OOV III, p. 325).

${ }^{81}$ La première partie du verset est traitée en (C22).

82 L'emploi du locatif pour l'objet direct est étonnant.
} 
Georges Rebuschi — Lapurdum 5 (2000) : Appendice

(C22) 7,14 Zer ersi da borta eta zer mear senda edo bidia eramaitan dionak akaba ez deiteken biziara! 'Qu'étroit et resserré est le chemin qui mène à la vie éternelle.'

(C23) 9,9 [...] ekusi zion gizon bat xaseririk [...] mai batian, izenez zionak Mateo, eta erran zaun [...] 'il vit un homme assis à une table, qui s'appelait Matthieu, et il lui dit' [lit. ...à une table, le-ERG-quiavait pour nom M.]

L'ex. suivant, (C24), pourrait par contre sembler régulier plutôt qu'exceptionnel, puisque l'on a une apposition à un nom propre à l'ergatif; mais, en fait, c'est l'ensemble baizen entregatan zaunak, relative appositive avec bait- comme $\mathrm{C}^{\circ}$, qui est en apposition, et qui contient la RSL nst. entregatan zaunak [basque standard entregatzen ziona], qui y a elle-même la fonction d'un attribut de copule : il s'agit donc bien à nouveau d'une RSL nst. irrégulière, qui ne répond pas aux contraintes distributionnelles usuelles.

(C24) 26,25 Eta artan ziolarik elia Judasek, baizen Ø entregatan zaunak, erran zion [...] 'Et quand Judas[ERG], qui était celui[ERG] qui le[DAT] livrait, prit la parole, il dit...'

Après la position prédicative, la position focale : c'est ce positionnement, et non la présence d'un quantificateur universel en lieu et place de l'article $(-a)$, question traitée à propos de (A17) et (A44) supra, qui représente une nouveauté en (C25), car la position non-argumentale occupée ici par la RSL nst. n'est pas celle d'un topique disloqué, mais celle d'un élément sous la portée focalisante d'un opérateur de négation, $e z$ :

(C25) 7,21 Ez niri erraiten dion orok “ $O$, Jeina, Jeina! ” sarten da Ø korrengatik zeurietako reinuan. 'Ce n'est pas celui, quel qu'il soit, qui me dit "Seigneur, Seigneur!" qui entre pour autant dans le royaume des cieux ' lit. 'NEG tout[ERG] qui me dit "S., S.! " entre[INAC] dans...'

Quant à (C26), c'est l'unique exception que j'aie relevée à travers les textes examinés comportant un antécédent explicite - à gauche de la relative, $\mathrm{cf}$. $(5 \mathrm{c}, \mathrm{d})$ du texte principal, mais cela ne change rien à l'affaire.

(C26) 13,39 Enemigo erin zionak da diabiua 83 'L'ennemi qui l'a semé, c'est le Diable.'

L'avant dernier exemple est une copie conforme de ce qui se passe en (A13-16) : on s'y reportera donc pour les explications.

(C27) 5,41 eta eramanaraztan daizunari [... ] milla urratzez, fan zte arekin berze bi millaz. 'Et (à] celui qui vous oblige à faire mille pas, faites-en [lit. 'allez'] avec lui deux mille [lit. 'autres']'

Enfin, le dernier exemple, conservé pour la bonne bouche, est aussi le dernier qui apparaisse chez Hualde, et celui qui pose le plus de problèmes. En voici d'abord une première version, légèrement tronquée :

(C28) 26,48 nik bixiko emoitan duanari, kori da: asegura zazei.

moi-ERG baiser donnant AUX- $n$-SG-DAT lui est...

'Celui à qui je donne[rai] un baiser, c'est lui : saisissez-le.'

Le sens est clair, et permet d'identifier l'aux. comme la forme relativisée de $d u d$, dur, l'équivalent du b. standard $d u t$ à abs. 3 sg., et erg. 1 sg. : ce qui « manque», c'est, comme il est usuel dans tous les dialectes orientaux (et pas seulement ceux parlés en France), la marque de datif. Mais cette marque n'est en fait absente que si un élément nominal ou pronominal explicite figure dans la proposition (la relative nik bixiko emoitan du[d]an), ce qui n'est précisément pas le cas ici. On pourrait donc penser que l'on a dans cet extrait un indice très fort en faveur de l'hypothèse du déplacement de l'opérateur relatif abstrait porteur de la marque de cas de spéc, CP vers Spéc,DP, d'où ensuite, par accord entre la tête et le spécificateur, l'apparition du suffixe de datif à la fin de la RSL. En tout état de cause, cette analyse est nettement plus satisfaisante que celle de l'emploi du datif comme marqueur de topicalisation par défaut, dont on a bien vu qu'elle relève d'un

83 Curieusement, ou très significativement (?), le texte de BOE propose ziona, et ne note qu'en bas de page que l'original, tout comme l'éd. d'Urquijo (et, j'ajouterai, celle d'Estornes Lasa), avaient zionak. Du point de vue l'analyse, on ne peut utiliser cet hapax, me semble-t-il, pour défendre la thèse de VergnaudKayne, cf. la note (18) du texte principal. 
facteur textuel, et non phrastique (cf. (C27) et ses équivalents au § 1), inapplicable ici. Mais il y a un "mais"; la phrase complète est en effet la suivante :

(C28') 26,48 Zointa(n) nik bixiko emoitan duanari, $\quad$ kori da $\ldots$

quel-LOC moi-ERG baiser donnant AUX- $n$-SG-DAT lui est...

où la relative semi-libre est non seulement introduite par un relatif explicite, zointan $^{84}$, mais encore un relatif explicite marqué pour un cas tout-à-fait inattendu, le locatif! J'avoue ne pas comprendre du tout ce qui se passe ici ${ }^{85}$.

\section{Bilan}

Certains exemples ne sont simplement pas pertinents, en fait, comme (A13-16), ou (C27). Sur les 111 restants, on peut établir le décompte final suivant.

(a) On trouve 83 cas "normaux" de RSL nst. disloquées à gauche avec un ergatif relié à la position relativisée dans la subordonnée, le cas externe standard étant un absolutif, ou simplement non disponible, 7 RSL identiques à ceci près qu'elles occupent une position nonargumentale à droite de la matrice, et 2 RSL nst. au datif (au lieu d'un absolutif externe, attendu).

(b) Parmi les autres cas, on a:

6 RSL nst. à l'ergatif dont le cas externe, standard, aurait dû être le datif, et

5 RSL nst. à l'absolutif, alors que le marquage externe demandait l'ergatif.

(c), Enfin, les quelques cas restants sont nettement plus irréguliers, soit que la RSL soit en position indéniablement argumentale, soit que d'autres facteurs viennent perturber l'agencement casuel.

Etant donné le caractère artisanal de ce premier déblayage du terrain des RSL non-standard, je m'arrête ici, laissant à des gens mieux équipés que moi le soin de reprendre ce travail sur la base de corpus plus importants, et utilisant le cas échéant des critères de classement plus fins.

\footnotetext{
${ }^{84}$ Le texte d'Estornés Lasa (1982) donne bien zointan, mais la version présentée dans BOE, Erronkariera (1997) offre la forme zointa, qui est peut-être due à une troncation liée au fait que la consonne suivante est aussi un $\mathrm{n}$-.

85 Il en va de même avec l'extrait suivant d'Udabe (guip.) : Eta edozein itz egiten duena gizonaren semearen kontra, izango zaio barkatua, baña edozein itz egiten duenari Espiritu Santuaren kontra, ez da barkatua izango gizonai [sic], ez siglo onetan, ez ere datorrenean. 'Et celui[ABS], quel qu'il soit, qui parle contre le fils de l'homme, il lui[DAT] sera pardonné, mais à celui[DAT] qui parle contre l'Esprit saint, il ne sera pardonné à l'homme ni en ce siècle, ni dans le suivant' (Mt 12,34). On a apparemment d'abord un emploi injustifié de l'absolutif, puisque le prédicat externe demande le datif, et le prédicat interne, l'ergatif, et, dans la secionde partie, une reprise non par un pronom, mais par un DP à tête lexicale, gizona.
} 
Georges REBUSCHI — Lapurdum 5 (2000) - A propos d'une construction non-standard du basque

\section{BIBLIOGRAPHIE}

\section{Références du corpus}

AGUIRRE, Acisclo X., de -. 1812-14. Ms. de sermons, publiés dans BOE, Bizkaiera II (1992), 605-794, sous le titre 'Oñatiko sermoiak'.

AÑIBARRO, Pedro Antonio. \pm 1800 . Jesu Christoren lau Evangelioac batera alcarturic. Ms., éd. par M. P. Ciarrusta, Bilbao : Jarein, 1991.

[Anon. A]. El Santo Evangelio [...] segun San Mateo. Ms. du $19^{\mathrm{e}}$ siècle publié in BOE, Hegoaldeko Goi-nafarrera IV (1996), 1277-1341, sous le titre 'San Mateoren Ebanjelioa'.

[Anon. B]. Jesucristoren evangelio [sic] Santua San Mateoren araura. Ms. du milieu du $19 \mathrm{e}$ siècle, publié dans BOE, Gipuzkera IV (1993), 2101-2109 [le texte va seulement de 1,1 à 6,7].

[Anon. C]. $18^{\mathrm{e}}$ siècle. Trad. sans titre de Doctrina para la instrucción de la Familia de Casa en Bascuence sacada del Luz de la Fe [...] de J. Barón y Arín, publiée dans BOE, Iparraldeko Goi-Nafarrera II (1995), 687-988, sous le titre 'Familiaren instrukziorako Dotrina-1'.

AZKUE, R.M. [1942] Atsotizak. In : R. M. A., Euskalerriaren Yakintza (Madrid : Espasa-Calpe, $1959^{2}$ ), vol. III, 6-231.

BOE : voir Bonaparte Ondareko Eskuizkribuak.

BONAPARTE, Louis-Lucien. [1991]. Opera Omnia Vasconice. Bilbao : Euskaltzaindia [= rééd. en facsim. de tous les textes publiés par les soins par L.-L. B. au $19^{\mathrm{e}}$ siècle, sauf la Bible intégrale de Duvoisin].

Bonapaerte Ondarreko Eskuizkribuak. Bilbao: Univ. de Deusto (Deiker), 21 vol., sous la dir. de Rosa Miren PAGOLA et al., 1992-2000.

CAZENAVE [Casenave], \pm 1860 . Jesu Khristoin Ebanjelio Seidia San Mathiuïn aäbera. Ms., publié en 1999 dans BOE,Ekialdeko Behe-nafarrera I, 17-76.

DUVOISIN, Jean. 1859-65. Bible edo Testament Zahar eta Berria [...]. Londres. Facsim., Bilbao: Gran Enciclopedia Vasca, 1972.

EAB-1. 1983. Itun Berria - Elizen arteko Biblia. Imprimé à Lizarra (Navarre) : Bibli Elkarte Batuak.

ECHENIQUE, Bruno. 1855. S. Mateoin Evangelioa. Version "A": ms. éd. par P. Salaburu in: Baztango euskalkiaz, I (Bilbao: Univ. de Deusto, 1980), 35-124 [= version dite "A " dans $B O E$, Iparraldeko Goi-Goinafarrera, I (1995), 97-176]. Version "B" de $B O E$ : publiée à Londres en 1857, facsim. in OOV II, 91-151.

EGUIATEGUY, Jusef. 1785. Filosofo huskaldunaren ekheia. Francfort : B. Edelman. Rééd. annotée par T. Peillen, Bilbao: Euskaltzaindia, 1983.

EHEG. 1980. Itun Berria. Saint-Sébastien : Idatz (Herriko Elizbarrutietako Gotzaiak).

d'ETCHEPARE, B. 1545. Linguae vasconum primitiae; éd. critique par P. Altuna, Bilbao : Mensajero, 1980.

EUSKALTZAINDIA (ed.). 1991. Voir Bonaparte [1991].

HARANEDER, Joannes. 1740 [ms.]. Jesu Christoren Evangelio Saindua. Ed. par P. Altuna, Bilbao : Euskaltzaindia, 1990.

HARRIET, Maurice. 1855. Iesu-Christo gure Iaunaren Testament Berria [...]. Bayonne : E. Lasserre.

HUALDE-MAYO, Pedro Prudencio. \pm 1860 . Jesu-Kristo gore Jeinaren Ebanjelio Saintiua segun San Mateok, ms. éd.par J. Estornés Lasa, Fontes Lingva Vasconvm 39 (1982): 43-103. Aussi in BOE, Erronkariera (1997), 17-102.

HUALDE-MAYO, Pedro Prudencio. 1869. Le petit catéchisme espagnol du P. Astete, traduit en [...] roncalais, par Don Prudencio Hualde, curé de Vidangoz, vérifié et modifié sur les lieux mêmes par le Prince Luis-Lucien Bonaparte [... ]. Londres. Fac-sim : OOV III, 258-329. Ms. original publié dans BOE, Erronkariera (1997), 105-138.

IRIBARNEGARAY, X. \pm 1860 . Yesu Chrichtoin Ebanyeliua San Mathioin arabera. Ms., publié dans BOE, Mendebaldeko Behe-nafarrera (1999), 19-81. 
Georges REBUSCHI — Lapurdum 5 (2000) - A propos d'une construction non-standard du basque

IRIGARAY, Angel. 1958. Prosistas navarros contemporáneos en lengua vasca $\approx X X$-garren mendeko Nafarroako euskal idazleak. Pampelune: Institución Principe de Viana.

JUANCO, Juan Marco. 1834. Doctrina cristiana en bascuence [... ]. Ms., publié dans BOE, Zaraitzera (1997), 241- 261 sous le titre 'Otsagiko Dotrina'

KEREXETA, J. 1976. Euskal-Biblia (bizkaieraz). Bilbao : Bilboko Elizbarrutiko Gotzaintza.

LIZARRAGA, J, Joaquín (dit d'Elcano). 1868. Jesucristoren Evangelio Saindua, Juanec dacarran guisara. Londres. Rééd. fac-sim., OOV III, 13-102, \& éd. du ms. dans BOE, Hegoaldeko Goinafarrera IV (1996), 1235-1275.

LIÇARRAGUE, J. 1571. Iesus Christ Gure Iaunaren Testamentu Berria [...]. La Rochelle. Réimpr. en facsim. par Th. Linschmann \& H. Schuchardt, in : I. Leiçarragas Baskische Bücher von 1571 [...], Strasbourg: Trübner, 1900 ; facsim. de ce dernier, Bilbao :

Euskaltzaindia, 1990, 241-1197.

LOPEZ, Alphonsa. 1782. Guiristhino perfeccioniaren praticaren pparte bat Heuscarala itçulia [… A. Avignon: Aubanel.

"MANEZAUNDI". Voir ZUBIRI, Enrike.

MINONDO, Pedro J. 1869. Le petit catéchisme espagnol du P. Astete, traduit en [...] aezcoan, par Don Pedro José Minondo, Instituteur à Garralda [...], vérifié et modifié sur les lieux mêmes par le Prince Luis-Lucien Bonaparte [... ]. Londres. Facsim : OOV III, 258-329. Ms. original publié par I. Camino \& R. Gómez, 1992, ASJU 26.2, 453-581, et dans BOE, Aezkera (1997), 49-79, sous le titre "Garraldako dotrina-1".

MOG(U)EL, Juan Antonio. 1802. El catequista Bascongado / Cristau Eracasle Euscalduna. Ms. publié par A. Arejita, Bilbao : Jarein/Labayru, 1994. Aussi dans BOE, Gipuzkera IV (1993), 2411-2591.

OOV : voir BONAPARTE [1991]

OTAEG(U)I, Pedro. 18XX. San Mateoren Ebanjelioa Ceamaco escuera itzulia. Ms. publié dans BOE, Gipuzkera V (1993), 2799-2852.

d'OYHENART(E), Arnaud. 1657-1664. Proverbes et poésies basques. Rééd. trilingue sous la dir. de J.-B. Orpustan, Saint-Etienne-de-Baïgorry : Izpegi, 1992.

SALABERRY, M., dit d'Ibarrole. 1856. L'Evangile selon Saint Mathieu, sur la version de M. le Maistre de Sacy [...]. Bayonne: Veuve Lamaignère. Facsim., OOV III, 335-420.

SAMPER, Pedro José. 1869. Jesucristo gure Jaunaren Evangelio sandua segun San Mateo. Ms., publié en dans BOE, Zaraitzera (1997), 17-81, sous le titre 'San Mateoren Ebanjelioa'.

SATRUSTEGUI, J.M. 1981. 'Nueva contribución al fondo de textos antiguos vascos'. ASJU 15, $75-104$.

SOUBELET, Domingo. 1969. Jesus. Bayonne, sans indic. d'éditeur, imprimé à Vienne.

UBILLOS, Juan Antonio. 1785. Christau doctriñ berri-ecarlea [...]. Tolosa.

URIARTE, Jose Antonio. [ \pm 1860$]$. Salomonen proverbioetaco liburua. Ms. publié dans $B O E$, Gipuzkera III (1993), 765-804.

ZUBIRI, Enrike, dit "MANEZAUNDI". 1990. Artikulu bilduma [1928-1936]. Textes présentés et collectés par R. M. Pagola, Bilbao: Labayru. [Une autre sélection d'articles est publiée dans Irigaray 1958, 15-127.]

\section{Références linguistiques}

ARTIAGOITIA, Xabier. 1998. 'Determinatzaile sintagmaren hipotesia euskal gramatikan'. Uztaro 27, 33-61.

BACH, Emmon, \& COOPER, Robin. 1978. 'The NP-S Analysis of Relative Clauses and Compositional Semantics'. Linguistics and Philosophy 2.

BRESNAN, Joan, \& GRIMSHAW, Janet. 1978. 'The Syntax of Free Relatives in English'. Linguistic Inquiry 9.3, 331-391.

CHOMSKY, Noam. 1974. 'Questions of Form and Interpretation'. Montreal Working Papers in Linguistics 3, 1-42. Aussi in N.C., 1977, Essays on Form and Interpretation (Amsterdam : North-Holland), 25-59. 
Georges REBUSCHI — Lapurdum 5 (2000) - A propos d'une construction non-standard du basque

CHOMSKY, Noam. 1986. Knowledge of Language. New York : Praeger.

CHOMSKY, Noam. 1999. 'Derivation by Phase'. Ms., MIT.

CITKO, Barbara. (Sous presse.) 'Light-headed Relatives'. University of Pennsylvania Working

Papers in Linguistics.

DAYAL, Veneeta. 1997. Locality in WH Quantification. Dordrecht : Kluwer.

EGUZKITZA, Andolin. 1995. 'Adnominals in the Grammar of Basque'. In : J. Hualde \& J. Ortiz de Urbina (eds.), Generative Studies in Basque Linguistics (Amsterdam : Benjamins), 163187.

EUSKALTZAINDIA. 1999. Euskal gramatika; lehen urratsak, 5: Mendeko perpausak, 1. Bilbao : Euskaltzaindia

EVANS, Gareth. 1977. 'Pronouns, Quantifiers, and Relative Clauses', I \& II. Repris dans G.E., Collected Papers (Oxford : Clarendon, 1985), 76-175.

FREI, Henri. 1929. La grammaire des fautes. Genève.

GASON, J. et al. 1984. Abrégé de grammaire latine. Paris : Magnard.

GROOS, Anneke, \& van RIEMSDIJK, Henk. [1979] 1981. 'Matching Effects in Free Relatives : A Parameter of Core Grammar'. Theory of Markedness in Generative Grammar, Proceedings of the 1979 GLOW Conference (Pise : Scuola Normale Superiore), 171-216.

GROSU , Alex. 2000. 'The Semantic Diversity of Internally-Headed Relative Clauses'. Ms., Univ. de Tel Aviv.

HIRSCHBÜHLER, Paul, \& RIVERO, María Luisa. 1983. 'Remarks on Free Relatives and Matching Phenomena'. Linguistic Inquiry 14.3, 505-520.

KAYNE, Richard. 1994. The Antisymmetry of Syntax. Cambridge (Mass.) : MIT Press.

LAFITTE, Pierre. 1962. Grammaire basque (navarro-labourdin littéraire). Bayonne: Amis du Musée Basque \& Ikas.

LINK, Godehard. 1984 'Hydras : On the logic of relative constructions with multiple heads'. In J. Groenendijk et al. (eds.), Proceedings of the Fourth Amsterdam Colloquium (Dordrecht : Foris), 245-257.

MUJIKA, Jose Antonio. 1999. 'Erlatibo arrunta zehaztaile? Teoriak eta datuak'. Euskera 44.2, 1139-1158

OYHARÇABAL, Bernard. 1987. Etude descriptive de constructions complexes en basque [...]. Thèse d'Etat, Univ. Paris VII.

OYHARÇABAL, Bernard. 1988. 'Operatzaile isila euskarazko perpaus erlatiboetan'. ASJU 22.1, 93-97.

OYHARÇABAL, Bernard. 1989. 'Pro-drop and the Resumptive Pronoun Strategy in Basque'. In

L. Marácz \& P. Muysken (eds.), Configurationality. The Typology of Asymmetries

(Dordrecht : Foris), 63-83.

PARTEE, Barbara. 1987. 'Noun Phrase Interpretation and Type-Shifting Principles'. In J. Groenendijk, D. de Jong \& M. Stokhof (eds.), Studies in Discourse Representation Theory and the Theory of Generalized Quantifiers (Dordrecht : Foris), 115-143.

POSTAL, Paul. 1966. 'On So-called Pronouns in English'. In F. Dinneen (ed.), Monograph Series on Languages and Linguistics 19 [Georgetown Univ. Press]. Aussi in D. Reibel \& S. Schane (eds.), 1969, Modern Studies in English (Englewodd Cliffs, N.J. : Prentice-Hall), 201224 ; et in R. Jacobs \& P. Rosenbaum (eds.), 1970, Readings in English Transformational Grammar (Walton, Mass. : Ginn), 56-82.

PRINCE, Ellen. 1998. 'On the Limits of Syntax, with Reference to Left-Dislocation and Topicalization'.Syntax and Semantics 29 [ $=$ P. Culicover \& L. McNally, eds., The Limits of Syntax, Academic Press], 281-302.

QUIRK, Randolph, et al. 1972. A Grammar of Contemporary English. Londres : Longman.

REBUSCHI, Georges. 1998a. 'Nouvelles remarques sur haina'. Lapurdum 3, 53-75.

REBUSCHI, Georges. 1998b. 'Vie et mort d'un pronom de type C'. Exposés au Séminaire de syntaxe avancée (Paris VII/ParisVIII), janvier \& mars. Ms., U. Paris III. 
Georges REBUSCHI — Lapurdum 5 (2000) - A propos d'une construction non-standard du basque

REBUSCHI, Georges. 1999. 'Types de langues de types de constructions : le cas des corrélatives'. In A. Söres \& C. Marchello-Nizia (eds.), Typologie des langues, universaux linguistiques [Univ. Paris X: LINX, numéro spécial], 55-72.

REBUSCHI, Georges. 2000. 'Basque Left-Dislocated (Semi-)Free Relatives and Correlatives'. Conférence à la Table ronde sur la syntaxe et la sémantique des constructions relatives, Univ. de Tel Aviv, 14-16 juin.

REBUSCHI, Georges. (Sous presse.) 'Sur les relatives libres et les corrélatives du basque'. In M. Aurnague \& M. Roché (dir.), Romania et Vasconia. Hommage à Jacques Allières (Toulouse).

RIZZI, Luigi. 1997. 'The Fine Structure of the Left Periphery'. In L. Haegeman (ed.), Elements of Grammar ; Handbook of Generative Syntax (Dordrecht : Kluwer), 281-337.

SHIMOYAMA, Junko. 1997. 'Internally-Headed Relative Clauses in Japanese and E-Type Anaphora'. Ms., Univ. du Massachusetts à Amherst.

VAN RIEMSDIJK, Henk. 1997. 'Left Dislocation'. In E. Anagnostopoulou et al. (dir.), Materials on Left Dislocation (Amsterdam : Benjamins), 1-10.

VAN RIEMSDIJK, Henk. 2000. 'Free Relatives'. "Projet SYNCOM", ch. 44.

VERGNAUD, Jean-Roger. 1985. Dépendances et niveaux de représentation en syntaxe. Amsterdam : Benjamins.

ZWART, Jan-Wouter. 1998. 'Where is Syntax ? Syntactic Aspects of Left Dislocation in Dutch and English'. Syntax and Semantics 29 [=P. Culicover \& L. McNally, eds., The Limits of Syntax, Academic Press], 365-393. 